\title{
Active Vibration Control and Coupled Vibration Analysis of a Parallel Manipulator with Multiple Flexible Links
}

\author{
Quan Zhang, ${ }^{1}$ Chaodong Li, ${ }^{1}$ Jiantao Zhang, ${ }^{1}$ and Jiamei Jin ${ }^{2}$ \\ ${ }^{1}$ School of Mechatronic Engineering and Automation, Shanghai University, Shanghai, China \\ ${ }^{2}$ State Key Laboratory of Mechanics and Control of Mechanical Structures, Nanjing University of Aeronautics and Astronautics, \\ Nanjing, China
}

Correspondence should be addressed to Quan Zhang; lincolnquan@shu.edu.cn

Received 22 June 2016; Accepted 15 September 2016

Academic Editor: Matteo Aureli

Copyright (C) 2016 Quan Zhang et al. This is an open access article distributed under the Creative Commons Attribution License, which permits unrestricted use, distribution, and reproduction in any medium, provided the original work is properly cited.

\begin{abstract}
This paper addresses the active vibration control and coupled vibration analysis of a planar parallel manipulator (PPM) with three flexible links. Multiple piezoelectric ceramic transducers are integrated with the flexible links to constitute the smart beam structures, and hence the vibration of the flexible link can be self-sensed and self-controlled. To prevent the spillover phenomena and improve the vibration control efficiency, the independent modal space control combined with an input shaper is developed to suppress both the structural and the residual vibration of the flexible links. The coupled vibration features between rigid and elastic motions and the interaction effects among three flexible links are theoretically analyzed based on the one-pass rigid-flexible dynamic models. Numerical simulation and experiment results show that the vibration of the three flexible links is coupled through the moving platform and the vibration suppression efficiency is getting improved with the number of controlled flexible links increased.
\end{abstract}

\section{Introduction}

With the increasing demands for productivity in many industrial fields, such as semiconductor manufacturing, medical application, and automatic microassembly, parallel robots with light components are designed to fulfill the requirements of high speed and high accuracy. Lighter and more compact than their serial bulky counterparts, the flexible parallel robots are able to realize the desired trajectories with less time and higher precision. Such robots feature both the advantages of light part and parallel structure, for example, lower energy consumption, high speed and acceleration, higher kinematic precision, and greater load bearing capacity [1]. However, for the task of fast trace tracking and positioning which require high acceleration and deceleration, the unwanted vibrations are introduced to the manipulator due to the use of light components. In this situation, the settling times get longer and the positioning accuracy is decreased due to the residual vibration after the motion stop, which is countered to our initial goal. Furthermore, the structural vibration arising from the inertial force during the operation not only will decrease the precision of the trajectory tracking, but also will lead to system instability for certain configurations. Hence, the vibration suppression of such flexible manipulators has received much attention in recent decades $[2,3]$.

Active vibration control is an effective way to suppress both the structural and the residual vibration. Compared with the passive vibration control, it has better efficiency and a more advanced control method can be adopted. Active vibration control can be divided into the following two methods according to whether the additional vibration sensors and actuators are required: (1) suppressing the vibration based on the joint motion, for example, input shaping control and singular perturbation control, and (2) adding vibration sensors and actuators to form a closed-loop control system; thus, the functions of self-sensing and self-control of the flexible structure can be achieved, and a variety of modern control approaches are easily adopted to realize vibration attenuation with different requirement of the system. The input shaping control, as a feedforward vibration control method, is first to conduct the convolution between the input signals and a series of pulse signals, and then the 
shaped results are transferred to the robot/manipulator as new driving signals to suppress the unwanted vibration at the feedforward loop. Since Singer [4] proposed the input shaping method for vibration attenuation in the late 1980 s, such method has been widely applied in many serial structure manipulators $[5,6]$. Cole and Wongratanaphisan [7] proposed a general approach to input shaping with finite impulse response (FIR) filters, and the corresponding controllers were verified experimentally on a flexible link planar manipulator. To achieve motion tracking and vibration suppression of a flexible manipulator simultaneously, an optimal input shaping feedforward controller combined with an LQR feedback motion controller is developed by Deng et al. [8]. However, relatively little work has been published on the control of flexible parallel manipulators. Using an input shaper and multimode adaptive positive position feedback (PPF), the vibration of a two-flexible-link parallel manipulator in the maneuver motion was avoided by shaping the input command, and the residual vibration was attenuated through the PPF control [9]. Through locally linearized dynamic model of a parallel manipulator, Kozak et al. [10] applied the standard input shaping techniques to a two-degree-of-freedom (DOF) parallel manipulator, but only the simulation results were given. To suppress the residual vibration of a 3-DOF flexible planar parallel manipulator, $\mathrm{Li}$ et al. [11] compared three different input shapers, namely, the zero-vibration (ZV) shaper, the zero-vibration-derivative (ZVD) shaper, and the extra insensitive (EI) shaper, in order to obtain better suppression results. In our previous work [12], a ZVD input shaper was incorporated with the motion controller to suppress the residual vibration of a 3-PRR parallel manipulator with three flexible links. However, due to the highly coupled dynamic characteristic of the 3-PRR rigidflexible manipulator, the ZVD input shaper can only suppress the unwanted vibration of the flexible links to some extent. Thus, to enhance the vibration suppression effects, the active vibration control laws based on the collocated piezoelectric sensors and actuators are developed to be incorporated with the input shaper in this study.

The piezoelectric transducers, as a kind of smart material possessing the characteristics of direct piezoelectric effect and inverse piezoelectric effect, have the ability to be both the vibration sensors and the actuators. During the control process, the vibration response of the flexible structure is detected by the piezoelectric sensor according to the piezoelectric materials' direct piezoelectric effect, and then the vibration signals are amplified by the vibration controller. At last, the piezoelectric actuators are actuated by the resulting control voltages to realize the vibration suppression based on the inverse piezoelectric effect. A variety of vibration controllers are proposed based on the above closed-loop control principle. In [13], two innovative vibration control laws, namely, the $\mathrm{H}$-infinite modified $\mathrm{PPF}$ and the $\mathrm{H}$ infinite modified positive velocity feedback control method, are studied to suppress the multiple modes vibration of an aluminum cantilever beam mounted with lead zirconate titanate (PZT) transducers. Zhang et al. [14] conducted disturbance rejection control to suppress the unwanted oscillation excited by unknown disturbances of a thin-walled piezoelectric integrated smart structure. Combined with variable structural control (VSC) and direct output feedback control, a hybrid controller is developed to achieve both the motion tracking and the vibration control of a flexible parallel manipulator by Zhang et al. [1]. Using strain and strain rate feedback controllers, Zhang et al. [15] conducted the active vibration control of a high speed parallel manipulator with three flexible links. However, in the practice of active vibration control, usually only a small number of modes are chosen to be suppressed, and hence the uncontrolled modes may lead to spillover, a phenomenon in which control energy flows to the uncontrolled modes of the system. In this situation, the control laws based on the first several selected modes may excite the other residual modes, spoiling the vibration control performance [16].

To avoid the spillover and achieve separated modal control, the independent modal space control (IMSC) was first proposed by Meirovitch and Baruh [17]. Based on IMSC, Baz and Poh [18] developed modified independent modal space control (MIMSC) to suppress the vibration modes depending on the energy level to improve the control efficiency. Using an efficient modal control (EMC) method which requires low control voltages and simple feedback gains, Singh et al. [19] and Zhang et al. [20] suppressed multiple vibration modes of a cantilever beam and a 3-PRR flexible parallel manipulator, respectively. Based on IMSC, Wu et al. [21] experimentally studied the vibration control of the first three modes of a flexible cantilever beam. Derived from the relationship existing between the shape of the matrix and the possibility that the control forces are dissipative, a new approach to the synthesis of the modal controller is investigated by Braghin et al. [22]. The key step in modal feedback controller is to obtain the modal coordinates from the physical coordinates either through state observer or through modal filters. Integrated with a state observer and a disturbance estimator, Bagordo et al. [23] suppressed the vibration due to unknown disturbance forces in a large nonlinear flexible boom through IMSC. In [24], a single-input state observer is derived to estimate the modal displacements and velocities, and then the IMSC method is further adopted to attenuate the vibration of a multibody flexible boom driven by hydraulic actuators. To obtain the optimal actuator placement for vibration control in a truss-cored sandwich plate based on IMSC, Guo and Jiang [25] developed a state observer to identify the modal coordinates. To conduct the EMC approach, Zhang et al. [20] extracted the modal coordinates of a flexible beam in parallel manipulator using modal filters based on the relationship between the shape functions and the locations of three collocated piezoelectric sensors/actuators pairs. Compared with the state observer, modal filter has the advantages of exacting the modal coordinates independent of the control work and no observation spillover from the residual modes.

Due to the complex dynamic characteristic of the parallel manipulator with flexible links, coupled vibration occurs when the parallel manipulator moves at high speed. This means not only that the free vibration of the flexible links is excited, but also that many other forced vibration components are coupled with the natural frequency. These forced vibration frequencies are likely from the vibration 

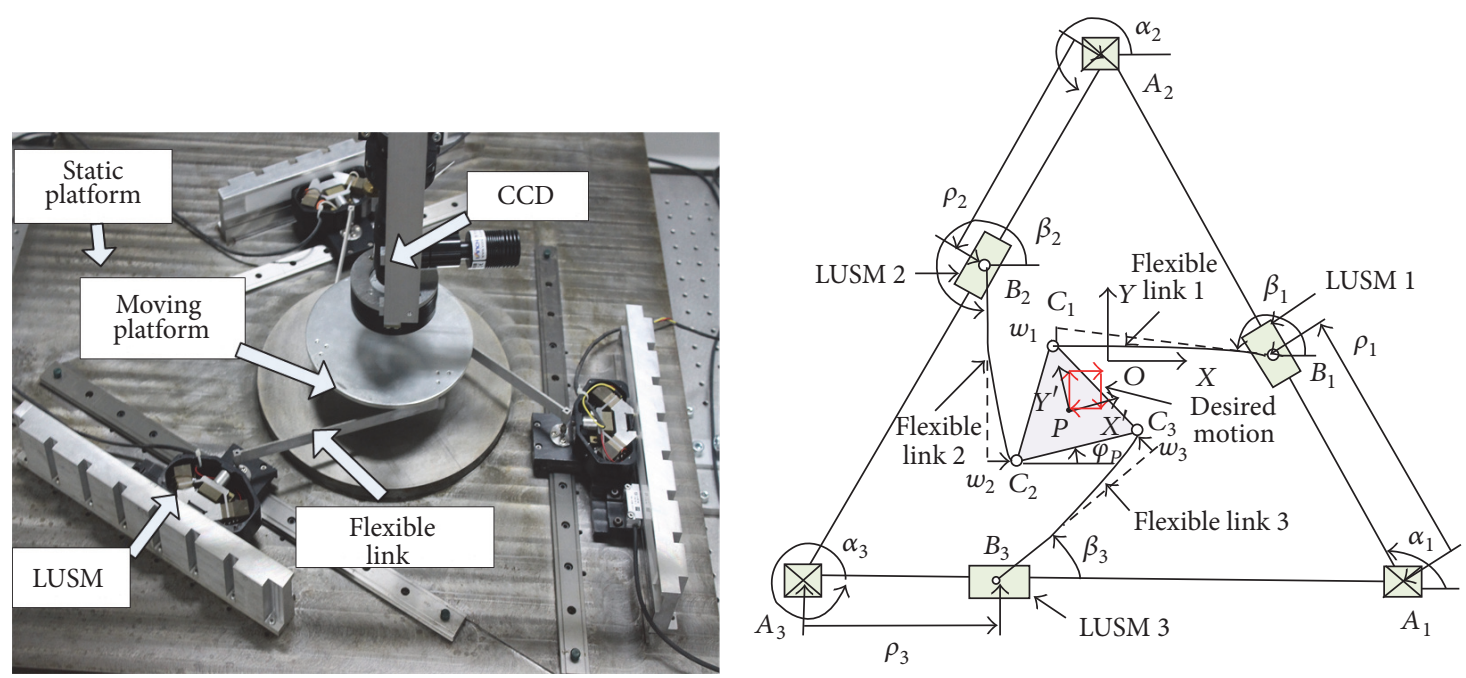

FIGURE 1: The prototype and mathematical model of the 3-PRR parallel manipulator.

of the driving motors, the inertial forces of the sliders, and the coupling effect between the rigid body motion and elastic motion. Furthermore, especially in the parallel manipulator with multiple flexible links, the vibration of the multiple flexible links is also coupled through the endeffector. Only suppressing one or two flexible links may amplify the vibration response of the other flexible links, which will further lead to the oscillation of the end-effector. Hence, to investigate such coupled vibration phenomenon and achieve the vibration suppression of multiple flexible links is the goal of this research. In our previous researches $[1,12,20]$, the active vibration suppression experiments are limited to only one flexible link and hence the control effects are restricted due to the coupled characteristic among the three flexible links. In this study, combined with input shaper and IMSC, active vibration control and coupled vibration features analysis of a parallel rigid-flexible manipulator with three flexible links are investigated both theoretically and experimentally. How coupled vibrations affect the rigid body motion is also discussed.

The rest of the paper is organized as follows. Section 2 describes the 3-PRR parallel manipulator with three flexible links and presents the one-pass dynamic model of the parallel manipulator. The design of IMSC method combined with input shaper is then developed in Section 3. In Section 4, the numerical simulation and theoretical analysis of the coupled vibration characteristics are studied. Section 5 presents the experiments results with different cases. Finally, conclusions are provided in Section 6.

\section{Dynamic Model of the Parallel Manipulator}

2.1. Introduction of the Flexible Parallel Manipulator Driven by LUSM. Linear ultrasonic motors, which utilize the converse piezoelectric effect and the ultrasonic vibration to output the linear motion directly, possess the advantages of high precision in the range of nanometers, no clearance from the transmission parts, and simple structure which can be easily integrated in the system. Hence, a 3-PRR type parallel manipulator with three light links driven by LUSMs is developed in our previous work. The prototype and the mathematical model are shown in Figure 1. The symbols are defined as follows: the layout angle of the three linear guides and the angle between the $x$-axis of the static frame and $i$ th links are defined as variable $\alpha_{i}$ and $\beta_{i}(i=1,2,3)$, respectively; the length of the linkage is represented by $L_{i}$; $\rho_{i}$ is the prismatic motion of the LUSM; the position of the end-effector in the static coordinates is stated as a vector of $\bar{X}_{p}=\left(x_{p}, y_{p}, \varphi_{p}\right)^{T} ; w_{i}\left(x_{i}\right)$ is the elastic deformation at $x_{i}\left(0 \leq x_{i} \leq L_{i}\right)$ of the $i$ th flexible linkage.

\subsection{Dynamic Model of the Rigid-Flexible Parallel Manipulator.} Dynamic model of the rigid-flexible parallel manipulator is a fundamental but significant work for the motion control and vibration suppression. The main modeling approach of such flexible parallel manipulator can be classified into two ways, namely, the two-pass method and the one-pass method. For the two-pass method, the rigid-flexible manipulators are first modeled as fully rigid body in order to calculate the inertial and joint forces, and then the prescribed rigid motion can be applied to the elastic model as excitation forces. Hence, in such method, for example, the KinetoElasto-Dynamics (KED) approach, only the effect of the rigid body motion on flexible body motion is considered. However, in the parallel manipulator with multiple flexible links, the vibration of the flexible links will also lead to the oscillation of the end-effector, decreasing the accuracy of the rigid motion, even causing system instability. Therefore, the effect of the elastic motion on rigid motion and also the coupled vibration among the multiple flexible links cannot be negligible. Thus, the one-pass method which considered the fully coupled dynamics characteristics between the rigid and elastic motions is adopted.

The basic principle in the one-pass model of this study is to use Lagrange multipliers to incorporate the rigid motion and elastic motion. According to the Lagrange equations, the 
total kinetic energy and the potential energy of the flexible parallel manipulator are presented as

$$
\begin{aligned}
T= & \sum_{i=1}^{3} \frac{1}{2} \int_{0}^{L_{i}} \rho_{A_{i}} \dot{\bar{r}}_{i}^{2} d x+\sum_{i=1}^{3} \frac{1}{2} m_{\mathrm{Si}} \dot{\rho}_{i}^{2} \\
& +\frac{1}{2} m_{p}\left(\dot{x}_{p}{ }^{2}+\dot{y}_{p}^{2}\right)+\frac{1}{2} I_{p} \dot{\varphi}_{p}^{2}, \\
V= & \sum_{i=1}^{3} \frac{1}{2} \int_{0}^{L_{i}} E_{i} I_{i}\left(w_{i}^{\prime \prime}\right)^{2} d x, \\
\bar{r}_{i}= & {\left[x_{a i}+\rho_{i} \cos \alpha_{i}+x \cos \beta_{i}-w_{i}(x, t) \sin \beta_{i}\right] i } \\
& +\left[y_{a i}+\rho_{i} \sin \alpha_{i}+x \sin \beta_{i}+w_{i}(x, t) \cos \beta_{i}\right] j,
\end{aligned}
$$

where the first and the second items of (1) represent the kinetic energies of the flexible links and the sliders, respectively, and the last two items are the kinetic energy of the moving platform. Equation (2) is the potential energy only considering the deformation of the flexible links since the manipulator is operated in horizontal plane. Equation (3) is defined as the position vector of $x_{i}$ on the $i$ th flexible links. Variable $w_{i}(x, t)$ is the transverse displacement of the $i$ th flexible link, $m_{p}$ is the mass of the moving platform, $m_{\mathrm{Si}}$ is the mass of the sliders, $m_{p}$ is the mass of the moving platform, $I_{p}$ is the mass moment of the platform, $\rho_{A_{i}}$ is the mass per unit length of the $i$ th sliders, and $\left(x_{a i}, y_{a i}\right)$ is the coordinates of point $A_{i}$ in the static frame. The length $L_{i}$, thickness $t_{b}$, and mass $m_{b}$ of the flexible links are $200 \mathrm{~mm}, 2 \mathrm{~mm}$, and $0.011 \mathrm{~kg}$, respectively. The masses of the moving platform $m_{p}$ and the sliders $m_{\mathrm{Si}}$ are $0.25 \mathrm{~kg}$ and $0.15 \mathrm{~kg}$.

Before applying Lagrange equations, the elastic motions, namely, the transverse vibration variable $w_{i}(x, t)$, are required to be discrete. Hence, the assumed mode method is adopted as follows:

$$
w_{i}(x, t)=\sum_{j=1}^{r} Y_{i j}(x) q_{i j}(t) \quad i=1,2,3,
$$

where $q_{i j}(t)$ represents the generalized elastic motion coordinates of the $i$ th link and $Y_{i j}(x)$ are shape functions matching pinned-free boundary conditions which were verified in our previous modal test experiments [12]. Then, the Lagrange multiplier equation which is used to integrate both rigid and elastic motions is given as

$$
\frac{d}{d t}\left(\frac{\partial(T-V)}{\partial \dot{\bar{\eta}}}\right)-\frac{\partial(T-V)}{\partial \bar{\eta}}=\bar{Q}+\sum_{k=1}^{6} \lambda_{k} \frac{\partial G_{k}}{\partial \bar{\eta}},
$$

where $\bar{\eta}=\left[\begin{array}{llll}\bar{\rho} & \bar{\beta} & \bar{X}_{p} & \bar{q}\end{array}\right]^{T} \in R^{(9+3 r) \times 1}$ is the generalized coordinates of the rigid-flexible manipulator, $\bar{q}=\left[\begin{array}{lllllllll}q_{11} & \cdots & q_{1 r} & q_{21} & \cdots & q_{2 r} & q_{31} & \cdots & q_{3 r}\end{array}\right]^{T} \in R^{3 r \times 1}$ is the generalized coordinates of the elastic motion, $\bar{Q}=$ $\left[\begin{array}{llllllllllll}F_{a 1} & F_{a 2} & F_{a 3} & F_{11} & \cdots & F_{1 r} & F_{21} & \cdots & F_{2 r} & F_{31} & \cdots & F_{3 r}\end{array}\right]^{T} \in$ $R^{(9+3 r) \times 1}$ represents the generalized forces applied by LUSM and piezoelectric vibration actuators, and $\lambda_{k}$ is the Lagrange multipliers. Particularly, $G_{k}$ is the closed-chain constraint equations which connected the rigid motions $\bar{\rho}, \bar{\beta}$, and $\bar{X}_{p}$ and the elastic motion $\bar{q}$. The detailed expression of $G_{k}$ is presented as

$$
\begin{array}{r}
x_{p}+x_{c i}^{\prime} \cos \varphi_{p}-y_{c i}^{\prime} \sin \varphi_{p} \\
=x_{a i}+\rho_{i} \cos \alpha_{i}+L_{i} \cos \beta_{i}-\sum_{j=1}^{r} Y_{i j}\left(L_{i}\right) q_{i j} \sin \beta_{i} \\
(i=1,2,3), \\
y_{p}+x_{c i}^{\prime} \sin \varphi_{p}+y_{c i}^{\prime} \cos \varphi_{p} \\
=y_{a i}+\rho_{i} \sin \alpha_{i}+L_{i} \sin \beta_{i}+\sum_{j=1}^{r} Y_{i j}\left(L_{i}\right) q_{i j} \cos \beta_{i} \\
(i=1,2,3) .
\end{array}
$$

From (5)-(6), it is clearly shown that the rigid and elastic motions are coupled through the geometric closed-chain constraint equations, and the elastic motion of the $i$ th flexible link is also coupled with other flexible links through the rigid motion, namely, through the moving platform.

System equations (5) are not in a suitable form for control synthesis since the Lagrange multiplier $\lambda_{k}$ is unknown and the rigid motions $\bar{\rho}, \bar{\beta}$, and $\bar{X}_{p}$ are not independent. Hence, the independent coordinate formulation [26] is employed to reduce the dimension of (5) to $3+3 r$ while eliminating the Lagrange multiplier $\lambda_{k}$. The $3+3 r$ independent variables are chosen as $\bar{X}_{p}=\left[\begin{array}{lll}x_{p} & y_{p} & \varphi_{p}\end{array}\right]^{T}$ and $\bar{q}=\left[\begin{array}{lllllllll}q_{11} & \cdots & q_{1 r} & q_{21} & \cdots & q_{2 r} & q_{31} & \cdots & q_{3 r}\end{array}\right]^{T}$. The resulting rigid-flexible dynamic model is given as

$$
\begin{gathered}
{\left[\begin{array}{cc}
M_{r r} & M_{f r} \\
M_{r f} & M_{f f}
\end{array}\right]\left[\begin{array}{c}
\ddot{\bar{X}}_{P} \\
\ddot{\bar{q}}
\end{array}\right]+\left[\begin{array}{cc}
C_{r r} & C_{f r} \\
C_{r f} & C_{f f}
\end{array}\right]\left[\begin{array}{c}
\dot{\bar{X}}_{P} \\
\dot{\bar{q}}
\end{array}\right]} \\
+\left[\begin{array}{cc}
0 & 0 \\
0 & K_{f}
\end{array}\right]\left[\begin{array}{c}
\bar{X}_{P} \\
\bar{q}
\end{array}\right]=\left[\begin{array}{c}
J_{P}{ }^{T} \bar{F}_{a} \\
J_{P w}{ }^{T} \bar{F}_{a}+F_{\mathrm{PZT}}
\end{array}\right],
\end{gathered}
$$

where the detailed expression of (7) and the system reduction procedure can be found in [1].

2.3. Coupled Dynamic Analysis of the Rigid and Elastic Motion. In order to facilitate the controller design, the dynamic model for rigid body and flexible body can be written in a separate form to facilitate the controller design and theoretical analysis:

$$
\begin{aligned}
M_{r r} \ddot{\bar{X}}_{P}+C_{r r} \dot{\bar{X}}_{P} & =J_{P}^{T} \bar{F}_{a}+\bar{T}_{d}, \\
M_{f f} \ddot{\bar{q}}+K_{f} \bar{q} & =\bar{F}_{\mathrm{PZT}}+\bar{t}_{d},
\end{aligned}
$$

where $\bar{t}_{d}=-M_{r f} \ddot{\bar{X}}_{P}-C_{r f} \dot{\bar{X}}_{P}+J_{P w}{ }^{T} \bar{F}_{a}-C_{f f} \dot{\bar{q}}$ is considered as the lumped disturbances of the flexible dynamics, $-M_{r f} \ddot{X}_{P}+$ $J_{P w}{ }^{T} \bar{F}_{a}$ represents the exciting forces applied to the elastic motion resulting from the rigid motion, and $-C_{r f} \dot{\bar{X}}_{P}-C_{f f} \dot{\bar{q}}$ is 


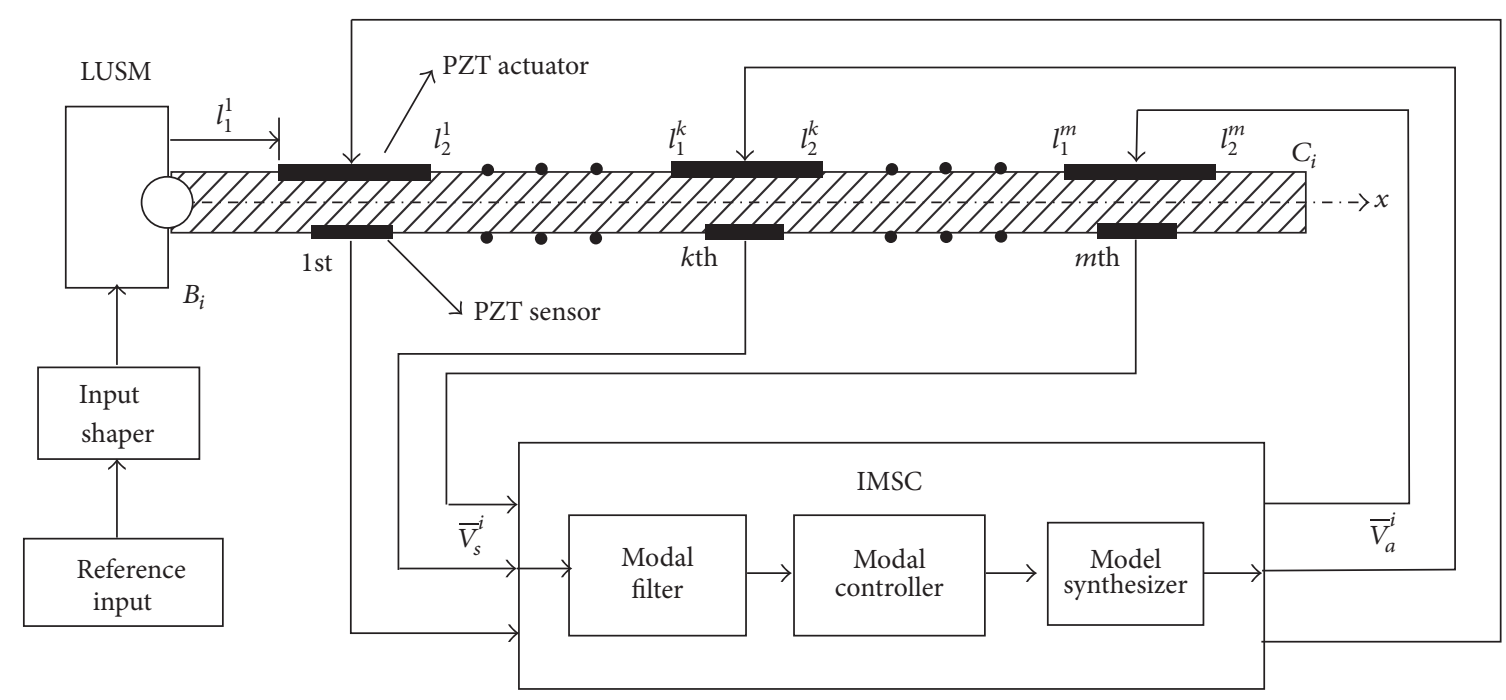

FIGURE 2: The proposed hybrid vibration control method.

the coupling forces due to the interaction between rigid and elastic motions. $F_{\text {PZT }}$ represents the vector of actuating force in state space converted from the strain generated by PZT actuator. $\bar{T}_{d}=-M_{f_{r}} \ddot{\bar{q}}-C_{f r} \dot{\bar{q}}$ is considered as the lumped disturbances arising from the rigid motion.

Equation (8) is a typical form for rigid body motion control with bounded disturbances, and hence variable modern control approaches can be adopted to actuate the LUSM, such as the robust variable structure control, computed torque control, and PD control. Since the controller design for the LUSM is not the main work of this study, the practical PD control is adopted to combine with the active vibration control method to investigate the coupled features of the rigid-flexible manipulator.

From (9), it can be clearly observed that the elastic motion of each flexible link is not only affected by the rigid body motion $-M_{r f} \ddot{\bar{X}}_{P}+J_{P w}{ }^{T} \bar{F}_{a}$, but also coupled with the deflection of other flexible links through the item $-C_{r f} \dot{\bar{X}}_{P}-C_{f f} \dot{\bar{q}}$. The corresponding matrices $M_{r f}$ and $C_{r f}$ are all configuration dependent, including both components of the rigid motion $\bar{X}_{p}$ and elastic motion $\bar{q}$. These coupled characteristics are also found in the system reduction procedure, since the closed-loop constraints equations (6), which are related to the motion of moving platform and vibration of the flexible links, are also used to eliminate the dependent coordinates $\bar{\rho}$ and $\bar{\beta}$.

\section{Vibration Controller Designs}

The design of an extra insensitive (ZV) input shaper integrated with IMSC method for vibration suppression of multiple flexible links is presented in this section. As shown in Figure 2, the basic principle of the hybrid controller is to combine the feedforward joint-based controller and the feedback PZT-based controller to attenuate both the structural and the residual vibration of the rigid-flexible manipulator.
3.1. Design of EI Input Shaper. Based on the input shaper techniques [27], a series of designed impulses are convolved with the input motion of the manipulator, and then the shaped signals are applied on the system at the corresponding time to eliminate the unwanted vibration modes. The commonly used input shapers can be classified as ZV shaper, ZVD shaper, ZVDD shaper, and extra insensitive (EI) shaper, and the main differences among these approaches are how to determine the impulses and the acting time with different conditions. Compared with other input shapers, the EI shaper increases the insensitivity to make the controller robust to the modeling error and disturbances by relaxing the zerovibration constraint. The vibration response of the flexible system for the shaped signals is given as [28]

$$
\begin{aligned}
& U(\omega, \varsigma) \\
& =e^{-\varsigma \omega t_{n}}\left\{\sum_{i=1}^{n}\left[A_{i} e^{-\varsigma \omega\left(t-t_{i}\right)} \cos \left(\omega \sqrt{1-\varsigma^{2}} t_{i}\right)\right]^{2}\right. \\
& \left.+\sum_{i=1}^{n}\left[A_{i} e^{-\varsigma \omega\left(t-t_{i}\right)} \sin \left(\omega \sqrt{1-\varsigma^{2}} t_{i}\right)\right]^{2}\right\}^{1 / 2}
\end{aligned}
$$

where $\omega$ represents the natural frequency and $\varsigma$ represents the damping ratio.

Then, the following constraint equations of EI input shaper are required to be satisfied:

$$
\begin{gathered}
U\left(\omega_{l}, \varsigma\right)=0 \\
U\left(\omega_{h}, \varsigma\right)=0 \\
U(\omega, \varsigma)=U_{t}, \\
\sum_{i=1}^{n} A_{i}=1 ; \\
\frac{\partial U(\omega, \varsigma)}{\partial \omega}=0
\end{gathered}
$$


where (11) means that, in order to maintain the symmetry of the system insensitivity, the vibration at two symmetric frequencies on opposite sides of the natural frequency is set to be zero, and the vibration at natural frequency is limited to less than a threshold $U_{t}$; hence, (12) guarantee the variation of the vibration at the natural frequency to be zero, and also the series impulses of $A_{i}$ are required to be normalized.

The impulse amplitudes and time locations for the single mode EI shaper are calculated from (11) and (12) as follows:

$$
\left[\begin{array}{c}
A_{i} \\
t_{i}
\end{array}\right]=\left[\begin{array}{ccc}
\frac{1+U_{t}}{4} & \frac{1-U_{t}}{2} & \frac{1+U_{t}}{4} \\
0 & \frac{\pi}{\omega \sqrt{1-\varsigma^{2}}} & \frac{2 \pi}{\omega \sqrt{1-\varsigma^{2}}}
\end{array}\right]
$$

3.2. Spillover Phenomenon Analysis. In order to conduct the closed-loop feedback control of the elastic motion of the flexible links, multiple pairs of PZT sensors and actuators are adopted to achieve the active vibration suppression, as shown in Figure 2. Based on the direct piezoelectric effect of the PZT material, when the flexible linkages are excited to vibrate, the strain is generated in the PZT sensor, and the resultant voltages can be obtained as the feedback vibration signal. Conversely, when a control voltage is applied to a PZT actuator, strain is generated on its surface due to the converse piezoelectric effect, and the equivalent control forces are achieved to suppress the unwanted vibration.

According to [29], the generalized modal control forces applied by PZT actuators corresponding to the modal coordinates $q_{i j}$ and the resultant voltages produced in the PZT sensors are given as

$$
\begin{aligned}
F_{\mathrm{PZT}}^{i j} & =\sum_{k=1}^{m} \lambda_{a}\left[Y_{i j}^{\prime}\left(l_{2}^{k}\right)-Y_{i j}^{\prime}\left(l_{1}^{k}\right)\right] V_{a k}^{i}(t), \\
V_{s}^{k}(t) & =\frac{b E_{p} d_{31} t_{e q}}{C_{p}^{l}}\left(\frac{\partial w\left(l_{2}^{k}, t\right)}{\partial x}-\frac{\partial w\left(l_{1}^{k}, t\right)}{\partial x}\right),
\end{aligned}
$$

where $\lambda_{a}$ is the constant of PZT actuator, $l_{2}^{k}$ and $l_{1}^{k}$ are the left and right end positions of the $k$ th $\mathrm{PZT}$ actuator, respectively, $V_{a k}^{i}(t)$ represents the control voltages imposed on the $k$ th PZT actuator of the $i$ th flexible link, $E_{p}$ is Young's modulus of PZT sensor, $d_{31}$ represents the constant of PZT materials, $b$ is the width of the PZT sensor, $C_{p}^{l}$ is the equivalent constant of piezoelectric capacitance, and $t_{e q}$ is defined as the distance between neutral axis of beam/sensor and that of midplane of beam and sensor.

However, the elastic motion has been discretized for $j$ th modes and practically the number of $j$ is finite; hence, usually only the first several dominant vibration modes are aimed to be controlled. In this situation, the controlled modes may excite the other residual modes and cause control energy flow to the uncontrolled modes. Using the strain rate feedback (SRF) control to give an example, according to (14) and (15) and the SRF control law, the applied control voltages corresponding to the response of the piezoelectric sensors are given as

$$
V_{a i}^{k}=-k_{d i}^{k} \dot{V}_{s i}^{k}=-k_{d i}^{k} \lambda_{s} \sum_{j=1}^{r}\left(Y_{i j}^{\prime}\left(l_{2}^{k}\right)-Y_{i j}^{\prime}\left(l_{1}^{k}\right)\right) \dot{q}_{i j}(t),
$$

where $\lambda_{s}=b E_{p} d_{31} t_{e q} / C_{p}^{l}$ and $k_{d i}^{k}$ represent the constant of the piezoelectric sensors and the positive feedback gains, respectively.

Substituting (16) into (14) and writing that in matrix form, the control forces of $k$ th piezoelectric actuator for $i$ th flexible links are given as

$$
{ }_{i}^{k} F_{\mathrm{PZT}}=-k_{d i}^{k} \lambda_{a} \lambda_{s}\left[\begin{array}{ccc}
\left(Y_{i 1}^{\prime}\left(l_{2}^{k}\right)-Y_{i 1}^{\prime}\left(l_{1}^{k}\right)\right)\left(Y_{i 1}^{\prime}\left(l_{2}^{1}\right)-Y_{i 1}^{\prime}\left(l_{1}^{1}\right)\right) & \cdots & \left(Y_{i 1}^{\prime}\left(l_{2}^{1}\right)-Y_{i 1}^{\prime}\left(l_{1}^{1}\right)\right)\left(Y_{i r}^{\prime}\left(l_{2}^{k}\right)-Y_{i r}^{\prime}\left(l_{1}^{k}\right)\right) \\
\vdots & \vdots & \vdots \\
\left(Y_{i r}^{\prime}\left(l_{2}^{k}\right)-Y_{i r}^{\prime}\left(l_{1}^{k}\right)\right)\left(Y_{i 1}^{\prime}\left(l_{2}^{1}\right)-Y_{i 1}^{\prime}\left(l_{1}^{1}\right)\right) & \cdots & \left(Y_{i r}^{\prime}\left(l_{2}^{k}\right)-Y_{i r}^{\prime}\left(l_{1}^{k}\right)\right)\left(Y_{i r}^{\prime}\left(l_{2}^{k}\right)-Y_{i r}^{\prime}\left(l_{1}^{k}\right)\right)
\end{array}\right]\left[\begin{array}{c}
\dot{q}_{i 1} \\
\vdots \\
\dot{q}_{i r}
\end{array}\right],
$$

where the constants of $\lambda_{a}$ and $\lambda_{s}$ are the same as other transducers since the $k$ th pairs of transducers are assumed to be identical. In real control experiments, the controller is developed based on the first several dominated modes for vibration suppression. If the first three modes are targeted for control in this study, then (17) can be partitioned and rewritten as

$$
{ }_{i}^{k} F_{\mathrm{PZT}}=\left[\begin{array}{l}
{ }_{i}^{k} F_{\mathrm{PZT}}^{3} \\
{ }_{i}^{k} F_{\mathrm{PZT}}^{n-3}
\end{array}\right]
$$

$$
\begin{gathered}
=-k_{d i}^{k} \lambda_{a} \lambda_{s}\left[\begin{array}{cc}
\phi_{3,3} & \phi_{3, n-3} \\
\phi_{n-3,3} & \phi_{n-3, n-3}
\end{array}\right]\left[\begin{array}{c}
\dot{q}_{i 1} \\
\vdots \\
\dot{q}_{i 3} \\
\dot{q}_{i 4} \\
\vdots \\
\dot{q}_{i n}
\end{array}\right] \\
=-k_{d i}^{k} \lambda_{a} \lambda_{s}\left[\begin{array}{c}
\phi_{3,3} \dot{\bar{q}}_{i, 3}+\phi_{3, n-3} \dot{\bar{q}}_{i,(n-3)} \\
\dot{\bar{q}}_{n-3,3}+\phi_{n-3, n-3} \\
\dot{\bar{q}}_{i,(n-3)}
\end{array}\right] .
\end{gathered}
$$


In (18), if the matrices $\phi_{3, n-3}$ and $\phi_{n-3,3}$ both happen to be zero, then the control forces are decoupled into modal form. But usually $\phi_{3, n-3}$ and $\phi_{n-3,3}$ are not zero, and hence the off-diagonal matrix causes the first 3 controller modes to be coupled with the residual $n-3$ modes. The item $\phi_{3, n-3} \dot{\bar{q}}_{i,(n-3)}$ represents the controlled modes affected by the excited residual modes, while the item $\phi_{n-3,3} \dot{\bar{q}}_{i, 3}$ is the control force flowing into the uncontrolled modes. Since the $n-3$ modes are not considered in the vibration control, the desired performance will be decreased. With the feedback gain $k_{d i}^{k}$ increasing, the flowing forces ${ }_{i}^{k} F_{\mathrm{PZT}}^{n-3}$ are amplified which will further excite the response of the residual modes. Then, the excited residual modes $\dot{\bar{q}}_{i,(n-3)}$ react on the controlled modes through the item $\phi_{3, n-3} \dot{\bar{q}}_{i,(n-3)}$. These unwanted cycles may cause system instability. Furthermore, for the three controlled modes, they are also coupled with each other since the matrix $\phi_{3,3}$ is off-diagonal. In conclusion, the interaction between controlled modes and uncontrolled modes is required to be avoided, and thus a goal can be achieved by designing the controller through independent modal control law.

3.3. IMSC Based on Piezoelectric Transducers. The basic principle of the IMSC is to control each mode independently in the modal space, with no coupling with other modes. The independent modal equation for the $j$ th mode of the $i$ th flexible link can be obtained from (9):

$$
\ddot{q}_{i j}+\omega_{i j}^{2} q_{i j}=\frac{\left(F_{\mathrm{PZT}}^{i j}+t_{d}^{i j}\right)}{m_{i j}}=f_{p}^{i j}+\widetilde{t}_{d}^{i j},
$$

where $\omega_{i j}$ reflects the modal frequency for the $j$ th mode of the $i$ th flexible link and $m_{i j}=\int_{0}^{L_{i}} \rho_{A_{i}} Y_{i j}{ }^{2} d x$ is a positive constant. Notice that the matrices of $M_{f f}$ and $K_{f}$ in (9) are all positive diagonal.

With IMSC approach, the modal force $f_{p}^{i j}$ is determined by amplifying the modal displacement and modal velocity as follows:

$$
f_{p}^{i j}=-k_{p}^{i j} q_{i j}-k_{d}^{i j} \dot{q}_{i j}
$$

Substituting (20) into (19) yields the closed-form equations for the $j$ th mode of the $i$ th flexible link as

$$
\ddot{q}_{i j}+k_{d}^{i j} \dot{q}_{i j}+\left(\omega_{i j}^{2}+k_{p}^{i j}\right) q_{i j}=\tilde{t}_{d}^{i j} .
$$

Compared with (19) and (21), it is clearly seen that the damping and the stiffness of the single mode are increased through applying the active control forces. The pole assignment method is adopted to determine the feedback gains of $k_{p}^{i j}$ and $k_{d}^{i j}$. Choose the pole position of the $j$ th controlled mode of the $i$ th flexible link as

$$
\bar{\gamma}_{i j}=-\bar{\alpha}_{i j}+j \bar{\beta}_{i j} \text {. }
$$

Notice that the relationship among mass, damping, stiffness, and the eigenvalues gives [23]

$$
\begin{aligned}
& k_{d}^{i j}=2 \bar{\alpha}_{i j}, \\
& k_{p}^{i j}=\bar{\alpha}_{i j}^{2}+\bar{\beta}_{i j}^{2}-\omega_{i j}^{2} .
\end{aligned}
$$

$k_{p}^{i j}$ and $k_{d}^{i j}$ determined by the pole assignment method are the initial values which need to be optimized to avoid the lower energy modes with higher control forces. In this study, the EMC method which uses the modal energy to modify the feedback gains is adopted [19]. The modified equation of $k_{p}^{i j}$ and $k_{d}^{i j}$ is shown below:

$$
\begin{aligned}
k_{p}^{i 1}: k_{p}^{i j}: k_{p}^{i r} & =k_{d}^{i 1}: k_{d}^{i j}: k_{d}^{i r} \\
& =1: \frac{E_{i j}}{E_{i 1}} \times \frac{\omega_{i 1}}{\omega_{i j}}: \frac{E_{i r}}{E_{i 1}} \times \frac{\omega_{i 1}}{\omega_{i r}},
\end{aligned}
$$

where $E_{i j}=\omega_{i j}^{2} q_{i j}^{2}+\dot{q}_{i j}^{2}$ reflects the modal vibration energy.

During the IMSC implementation, two key problems, namely, how to transfer the response voltages of the piezoelectric sensors to the modal coordinates and convert the determined modal forces to the applied voltages of the piezoelectric actuators, are required to be carefully processed. The first procedure can be achieved by a modal filter or a state observer while the latter may be obtained through a modal synthesizer. The detailed expression of the modal filter and modal synthesizer can be found in our previous work [20].

\section{Numerical Simulation}

This section presents the simulation results based on the rigid-flexible dynamic model and the proposed IMSC method. The Matlab/Simulink software is adopted to analyze the effectiveness of the active vibration control method and the coupled vibration characteristic among three flexible links. A square trajectory motion of the moving platform is chosen to perform the simulation, as the red trace illustrated in Figure 1. The square motion can be divided into four pointto-point motions with smooth acceleration and deceleration for each period, and the first point-to-point motion is given below:

$$
\begin{aligned}
y_{p}(t) & =\frac{y_{f}}{t_{f}} t-\frac{y_{f}}{2 \pi} \sin \left(\frac{2 \pi t}{t_{f}}\right), \\
x_{p} & =0, \\
\varphi_{p} & =\frac{\pi}{6},
\end{aligned}
$$

where $y_{f}=0.01 \mathrm{~m}$ and $t_{f}=0.1 \mathrm{~s}$.

The layout of the linear guide is an equilateral triangle $A_{1} A_{2} A_{3}$ with the length of $600 \mathrm{~mm}$, and the distance among the joint positions on the moving platform $C_{i}$ is $120 \mathrm{~mm}$. Notice that when one of the links is either vertical or parallel to its corresponding linear guide, the singular position of the manipulator will occur and the manipulator is uncontrolled. According to the initial configuration of the end-effector (namely, $\beta_{1}=\pi, \beta_{2}=(5 / 3) \pi$, and $\left.\beta_{3}=(1 / 3) \pi\right)$, the motion ranges of the three flexible links are restricted to $\beta_{1} \in((2 / 3) \pi,(7 / 6) \pi), \beta_{2} \in((4 / 3) \pi,(11 / 6) \pi)$, and $\beta_{3} \in(0,(1 / 2) \pi)$, respectively. During our trajectory tracking experiments, the motion ranges of the three links are calculated as $\beta_{1} \in((24 / 25) \pi, \pi), \beta_{2} \in((5 / 3) \pi,(17 / 10) \pi)$, and 

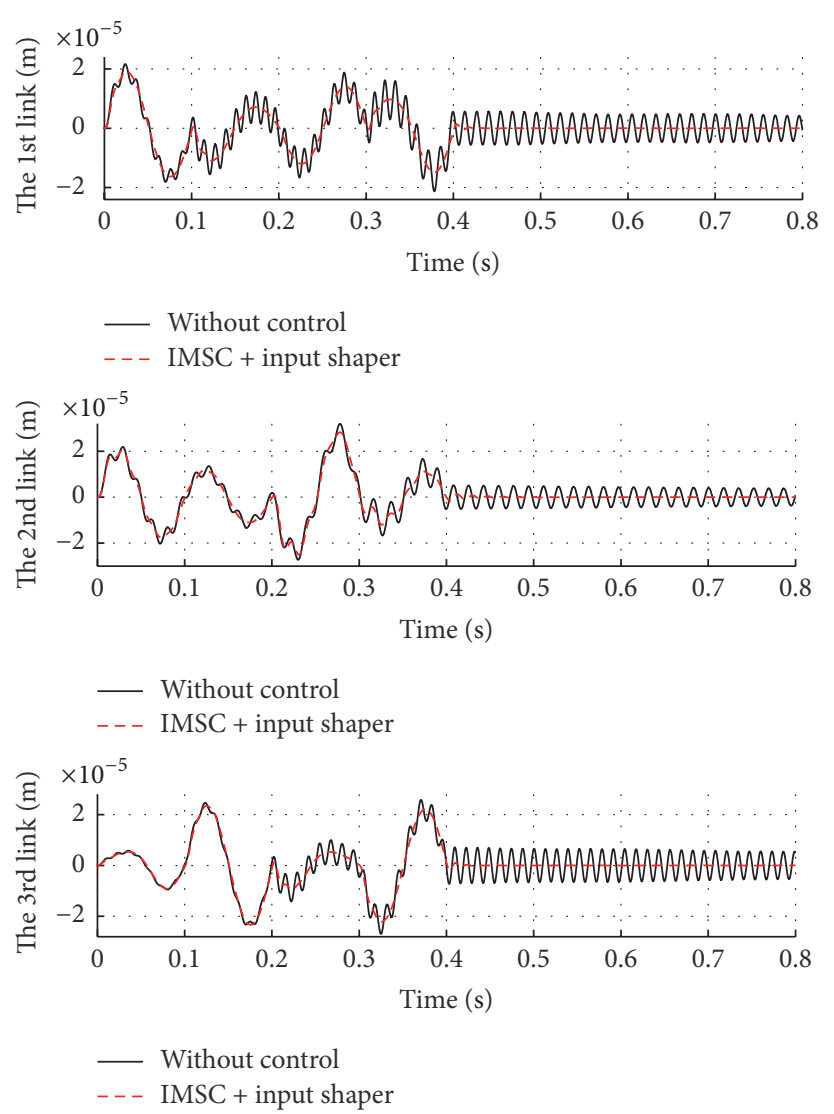

Figure 3: The vibration response at three-quarters of the three flexible links.

$\beta_{3} \in((1 / 3) \pi,(37 / 100) \pi)$, and hence the singular position of the manipulator can be avoided with the desired trajectory.

Three pairs of PZT transducers are mounted on each flexible link at the location of $50 \mathrm{~mm}, 100 \mathrm{~mm}$, and $150 \mathrm{~mm}$ as vibration sensors and actuators. The piezoelectric constant $d_{31}$, thickness $t_{p}$, and Young's modulus $E_{p}$ of the PZT transducers are $-180 \times 10^{-12} \mathrm{C} / \mathrm{N}, 0.2 \mathrm{~mm}$, and $6.6 \times 10^{10} \mathrm{~N} / \mathrm{m}^{2}$, respectively. In the simulation work, the first three modes of each flexible link are chosen to be suppressed. Since the main object is to damp the vibration, only the modal velocity feedback gains are determined to simplify the control procedure; namely, the modal position feedback gain $k_{p}^{i j}$ in (20) is supposed to be zero. Using the pole assignment method and the EMC method, the initial values of $k_{d}^{i 1}(i=$ $1,2,3)$ are calculated as $3.1,3.5$, and $3.4 \mathrm{~N} \cdot \mathrm{s} / \mathrm{m}$, and the modified ratios for the second and third mode of each flexible link are obtained from (24) as follows:

$$
\begin{aligned}
& k_{d}^{11}: k_{d}^{12}: k_{d}^{13}=1: 0.345: 0.045 \\
& k_{d}^{21}: k_{d}^{22}: k_{d}^{23}=1: 0.304: 0.041 \\
& k_{d}^{31}: k_{d}^{32}: k_{d}^{33}=1: 0.321: 0.038
\end{aligned}
$$

During the simulation process, the practical PD control [1] is adopted to actuate the rigid motion. To better validate the effectiveness of the proposed hybrid active vibration control method and the coupled vibration characteristic among the three flexible links, the vibration response of the system with three different cases with different number of flexible links controlled is investigated. Figures 3-6 are the simulation results under the first case when the vibrations of the three flexible links are suppressed simultaneously. As shown in Figure 3, it is clearly seen that both the structural and the residual vibration at the three-quarters $(150 \mathrm{~mm})$ of the three flexible links are effectively damped. Due to the different dynamic equations of each flexible link, the vibration responses of the three flexible links are not identical. Furthermore, since the feedback gains $k_{d}^{i j}$ are optimized by the uncontrolled vibration response energy, the modified ratios of three flexible links are also different from each other as shown in (26). Figure 4 reveals that the first three modes of the first flexible link (the three flexible links are numbered in Figure 1) are all attenuated effectively, which further verifies that the hybrid active vibration control method has the ability to suppress the multimode vibration simultaneously.

Figures 5 and 6 explained the coupled dynamic relationship between the rigid motion and the elastic motion. The position errors in $X, Y$, and $\varphi$ directions are illustrated in Figure 5. $\varphi$ is defined in Figure 1 which represents the rotation degree of freedom in the $X-Y$ plane. In the desired motion, $\varphi$ is a constant which equals $\pi / 6$. It can be found in Figure 5 that, during trajectory tracking period, namely, before $0.4 \mathrm{~s}$, the motion control error introduced by the PD 

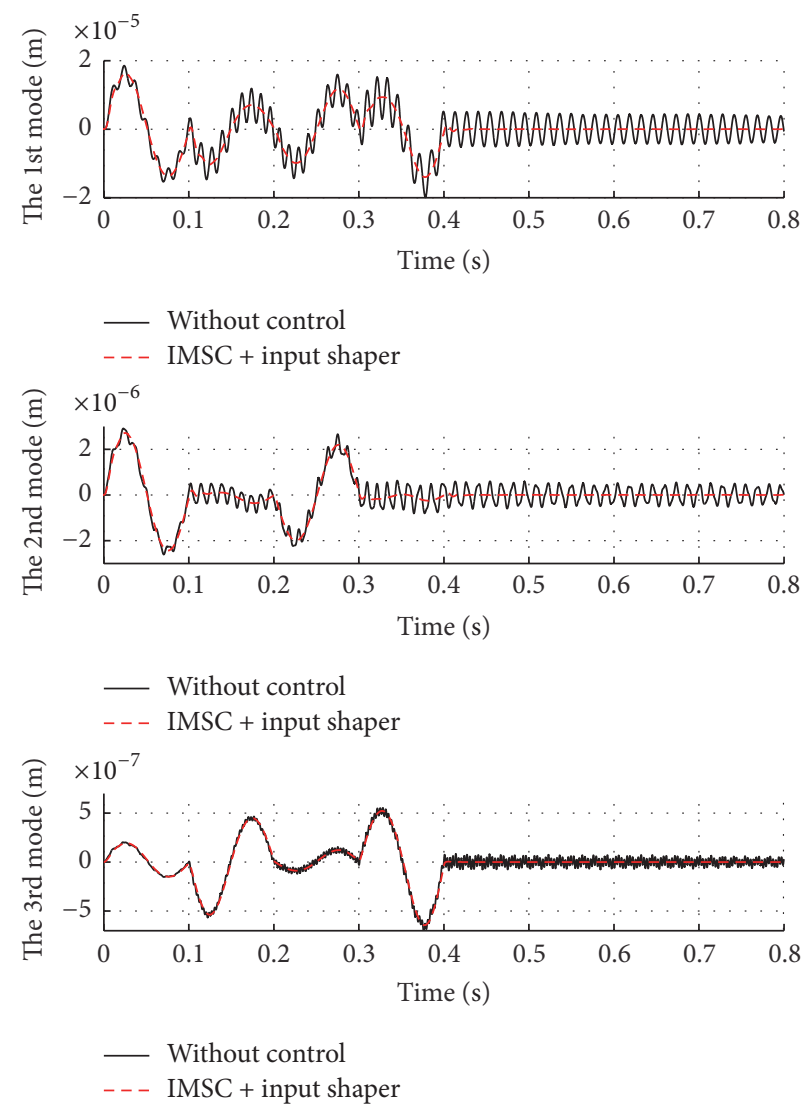

FIgURE 4: The first three modes' vibration response at three-quarters of the first links.

controller is the dominant error source, while, in the residual period, namely, after $0.4 \mathrm{~s}$, the oscillation error of the moving platform is mainly caused by the residual vibration of the three flexible links. Figure 5 also shows that the proposed hybrid active vibration control method has strong ability to suppress the residual oscillation error of the moving platform but has nearly no effect on the motion control error; this is mainly because the proposed vibration control method is only designed to damp the natural vibration mode of the flexible links which leads to the residual oscillation of the rigid motion. To better show the effect between the rigid and the elastic motion with and without the active vibration control method, the rigid motion response from $0.4 \mathrm{~s}$ to $0.8 \mathrm{~s}$ in Figure 5 is magnified 15 times in Figure 6 which clearly demonstrated that the residual oscillation errors in $X, Y$, and $\varphi$ directions are damped fast and effectively. This coupled rigid-flexible characteristic can be further explained theoretically by the rigid dynamic equation (8) in which the item $\bar{T}_{d}(\ddot{\bar{q}}, \dot{\bar{q}}, \bar{q})$ represents the elastic motion affected on the rigid motion. In (8), if the residual vibrations of the three flexible links are not damped effectively, the item $\bar{T}_{d}(\ddot{\bar{q}}, \dot{\bar{q}}, \bar{q})$ will keep affecting the rigid body motions as a continuous disturbance. Hence, when the vibrations of the three flexible links are suppressed, namely, $\ddot{\bar{q}} \rightarrow 0, \dot{\bar{q}} \rightarrow 0$, and $\bar{q} \rightarrow 0$, the item $\bar{T}_{d}(\ddot{\bar{q}}, \dot{\bar{q}}, \bar{q})$ is also attenuated to zero quickly, which will assist the rigid motion controller to stabilize the system in a very short time. Notice that since the item $\bar{T}_{d}(\ddot{\bar{q}}, \dot{\bar{q}}, \bar{q})$ can be recognized as a disturbance, the robust rigid motion control approaches, for example, the sliding mode control and the $H_{\infty}$ control, may be adopted to both damp the residual oscillation error and improve the motion tracking accuracy of the moving platform from the rigid motion's point of view.

To investigate the coupled vibration features among the three flexible links, the other two different control cases are conducted as comparisons. Figures 7 and 8 show the simulation results of the second case with only the first link under control. In this situation, as illustrated in Figure 7, both the structural vibration and the residual vibration of the controlled link are suppressed effectively, but the response of the other two links remains almost unchanged, except for a little variation in the residual vibration. The amplitude of the residual vibration of the second link exhibits a little decrease while the third link exhibits a little increase. It is observed in Figure 8 that the residual oscillation errors in $X, Y$, and $\varphi$ directions of the moving platform have been damped to some extent, but the effects are limited. Figures 9 and 10 show the simulation results of the third case with the first link and second link under control. In this case, the vibrations of the controlled links are suppressed as well as the first cases, and the vibration of the uncontrolled third link is also damped. Although the effect is still limited, the damped efficiency is better than that in the second case. Figure 10 shows that the residual oscillation errors in $X, Y$, and $\varphi$ directions of the moving platform are suppressed by more than $50 \%$, 

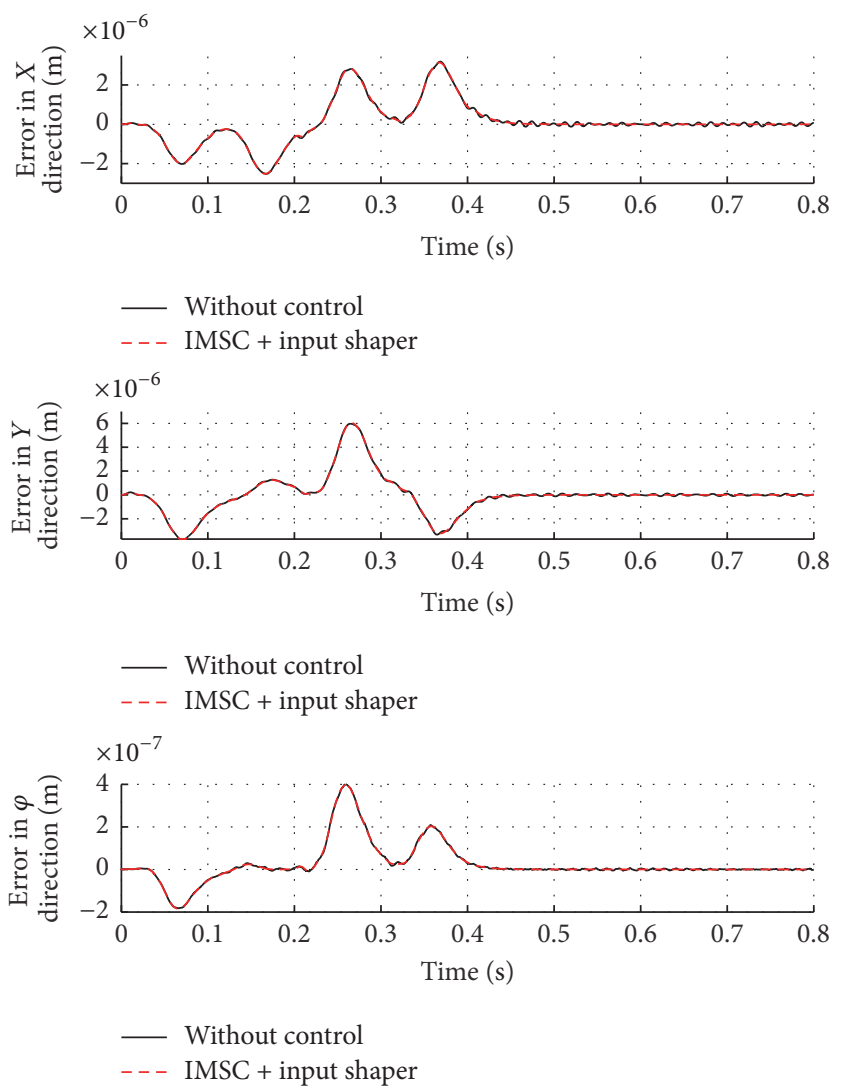

FIGURE 5: The positioning error of the rigid motion in 3 planar DOFs.
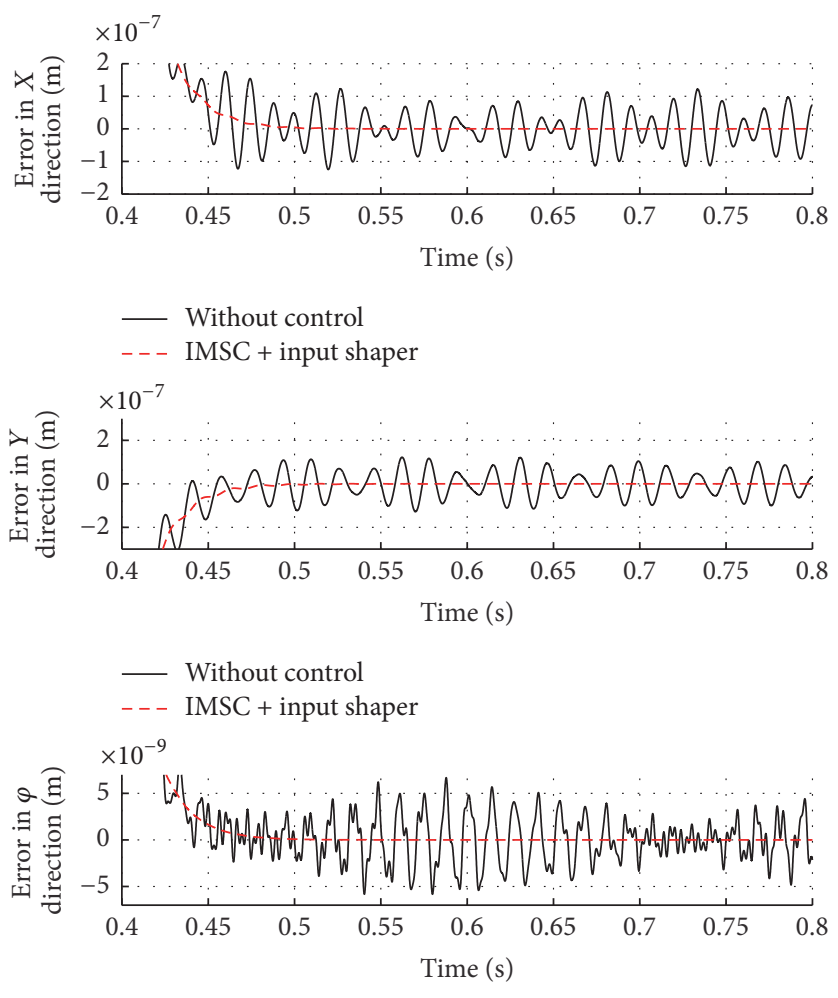

- Without control

- - - IMSC + input shaper

FIgURE 6: Amplified view of the positioning error of the rigid motion in 3 planar DOFs. 

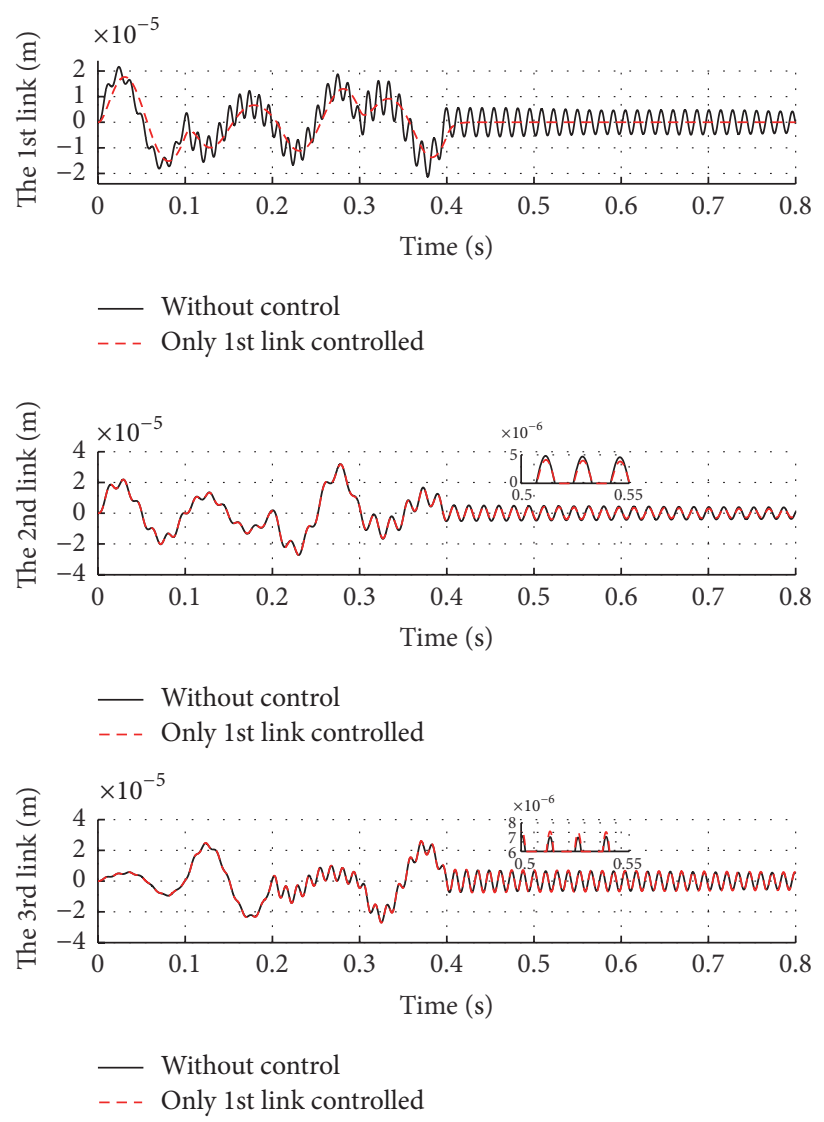

FIgURE 7: The vibration response of the three flexible links under the second case.

especially in $Y$ direction in which the residual oscillation has been almost attenuated. The phenomenon in Figures 7 and 9 can be theoretically analyzed through the elastic dynamic equation (9). When the trajectory tracking is finished and the flexible link is uncontrolled, the control vector $\bar{F}_{\mathrm{PZT}}$ and the first three components of item $\bar{t}_{d}=-M_{r f} \ddot{\bar{X}}_{P}-C_{r f} \dot{\bar{X}}_{P}+$ $J_{P w}{ }^{T} \bar{F}_{a}-C_{f f} \dot{\bar{q}}$ are all supposed to be zeros. Hence, the residual vibration of each link will be affected by other flexible links through the remaining item $-C_{f f} \dot{\bar{q}}$. Since the matrix $C_{f f}$ is not diagonal, the item $-C_{f f} \dot{\bar{q}}\left(\bar{q}_{1}, \bar{q}_{2}, \bar{q}_{3}, \dot{\bar{q}}_{1}, \dot{\bar{q}}_{2}, \dot{\bar{q}}_{3}\right)$ can be recognized as the interaction forces among the elastic motions. In the second and third cases, the elastic motions of the uncontrolled flexible links suffer the interaction forces of $-C_{f f} \dot{\bar{q}}\left(0, \bar{q}_{2}, \bar{q}_{3}, 0, \dot{\bar{q}}_{2}, \dot{\bar{q}}_{3}\right)$ and $-C_{f f} \dot{\bar{q}}\left(0,0, \bar{q}_{3}, 0,0, \dot{\bar{q}}_{3}\right)$, respectively, leading to a different vibration response in Figures 7 and 9. Notice that the more zero components the item $-C_{f f} \dot{\bar{q}}$ has, the less excited the vibration response is by the interaction forces. Figures 6, 8, and 10 also reveal that the more the flexible links are under control, the less excited the oscillation of the rigid body motion is through the item $\bar{T}_{d}\left(\ddot{\bar{q}}_{1}, \ddot{\bar{q}}_{2}, \ddot{\bar{q}}_{3}, \dot{\bar{q}}_{1}, \dot{\bar{q}}_{2}, \dot{\bar{q}}_{3}, \bar{q}_{1}, \bar{q}_{2}, \bar{q}_{3}\right)$.

\section{Experiment Results}

In this section, the experiment is conducted to validate the proposed hybrid active control method and the theoretical analysis of the coupled vibration characteristics. The motion control card (DMC-1842, GALIL) is used to control the three LUSMs through three LUSM drivers (made by NUAA). The feedback signal is provided by the linear grating sensor (LIA20, NUMERIK JENA). The DSP control boards (Seed DEC2812) are adopted to realize the active vibration control. A signal conditioner (NI SCXI 1531) and an actuation amplifier (SS08 Sensor Technology) are adopted to amplify the acquired voltage of the PZT sensors and the control voltages of the PZT actuators, respectively. Each flexible link is mounted with three pairs of PZT sensors and actuators (PZT5, CSSC) at the location of quarter, midpoint, and three-quarters, as shown in Figure 11. The dimensions of the sensor and the actuator are $10 \times 10 \times 0.2 \mathrm{~mm}$ and $30 \times 10 \times 0.2 \mathrm{~mm}$, respectively. Before the active vibration control experiments, fundamental tests are implemented to provide the corresponding data. Firstly, the modal testing experiment is carried out to obtain the natural frequency and the mode shapes of the flexible links. Based on the dynamic response analyzer, the first two mode shapes which are $92.5 \mathrm{~Hz}$ and $241.3 \mathrm{~Hz}$ and the mode shapes of pinned-free boundary conditions are validated [20]. Then, the calibration work is done to increase the control precision based on the particle swarm optimization method [12]. Finally, the vibration energy of the uncontrolled system for the desired trajectory is detected and calculated through the piezoelectric sensors and the modal filters. 

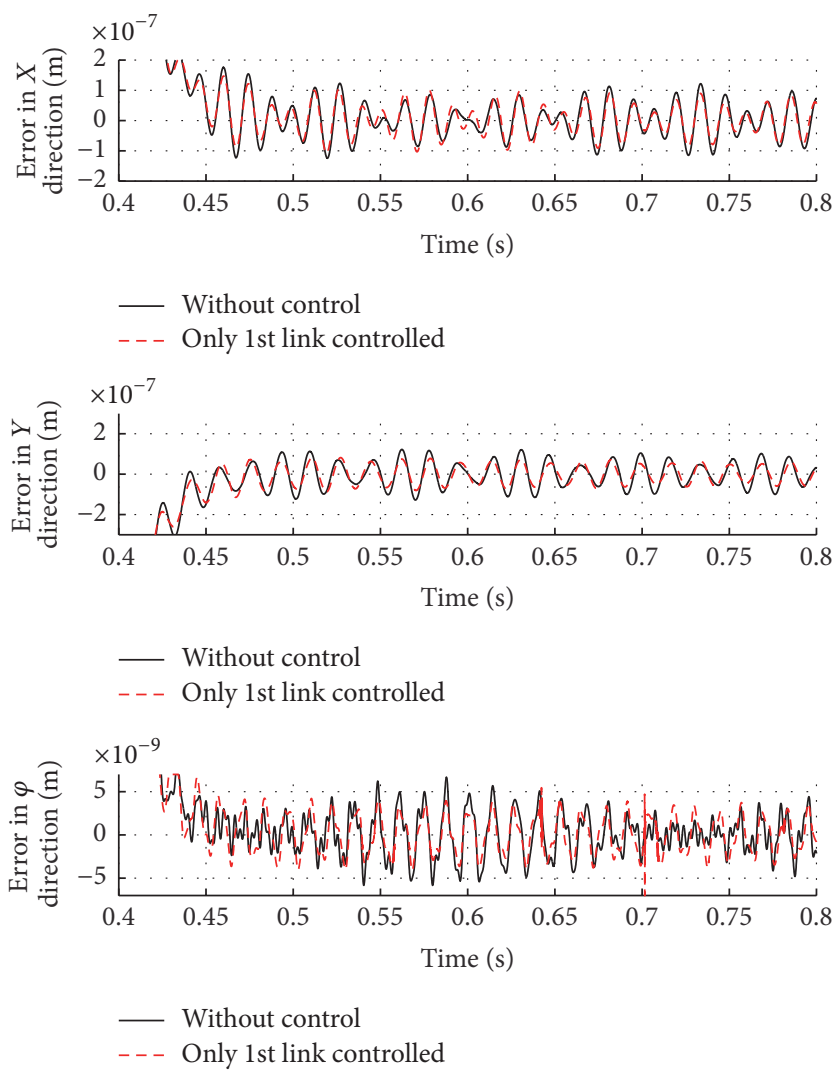

FIGURE 8: The positioning error of the rigid motion under the second case.

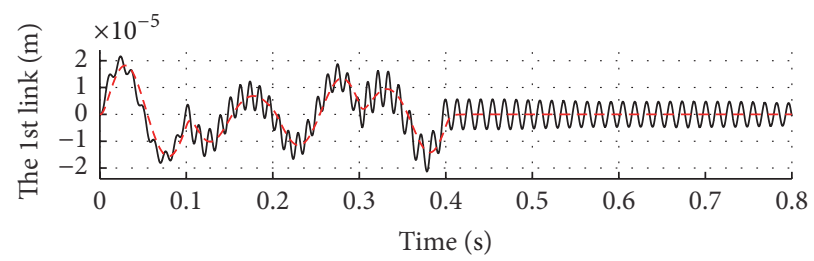

- Without control

- - Only 1st and 2nd links controlled

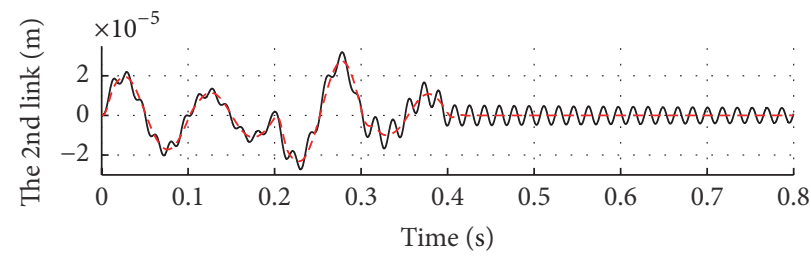

- Without control

- - Only 1st and 2nd links controlled

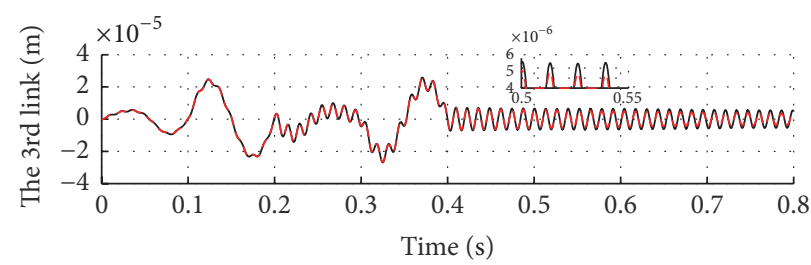

- Without control

- - - Only 1st and 2nd links controlled

FIGURE 9: The vibration response of the three flexible links under the third case. 

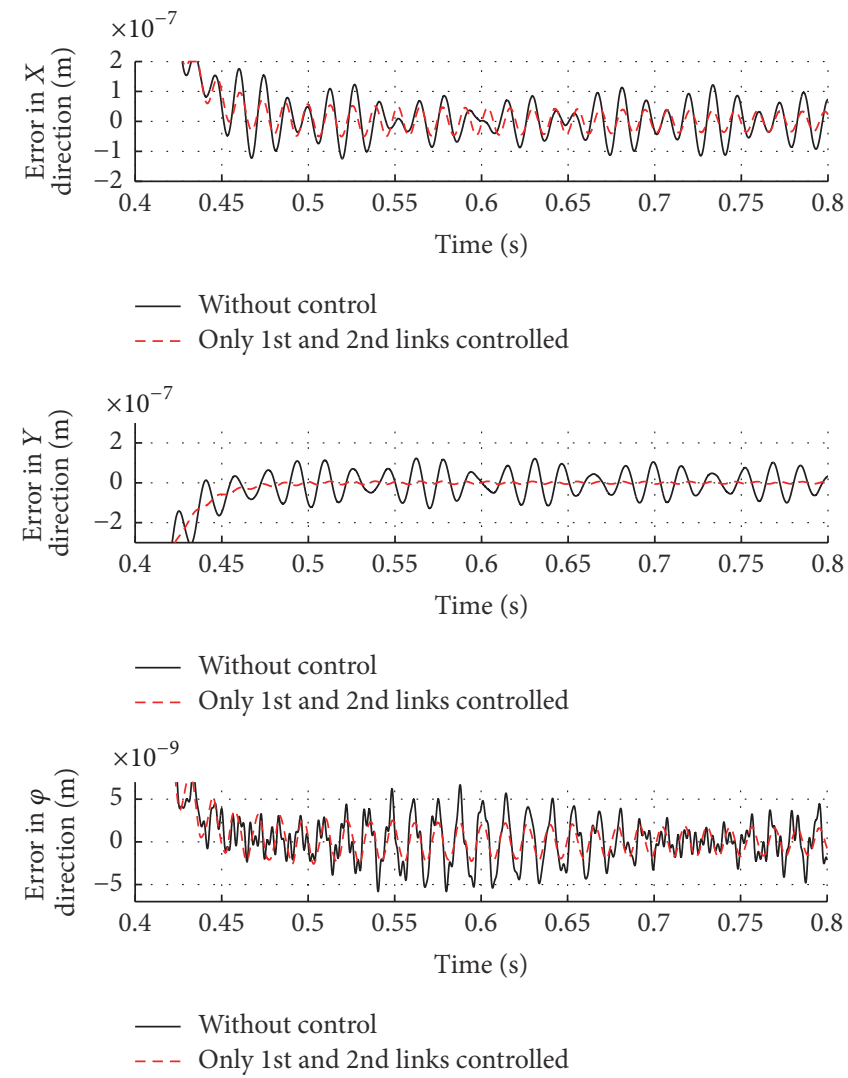

FIGURE 10: The positioning error of the rigid motion under the third case.

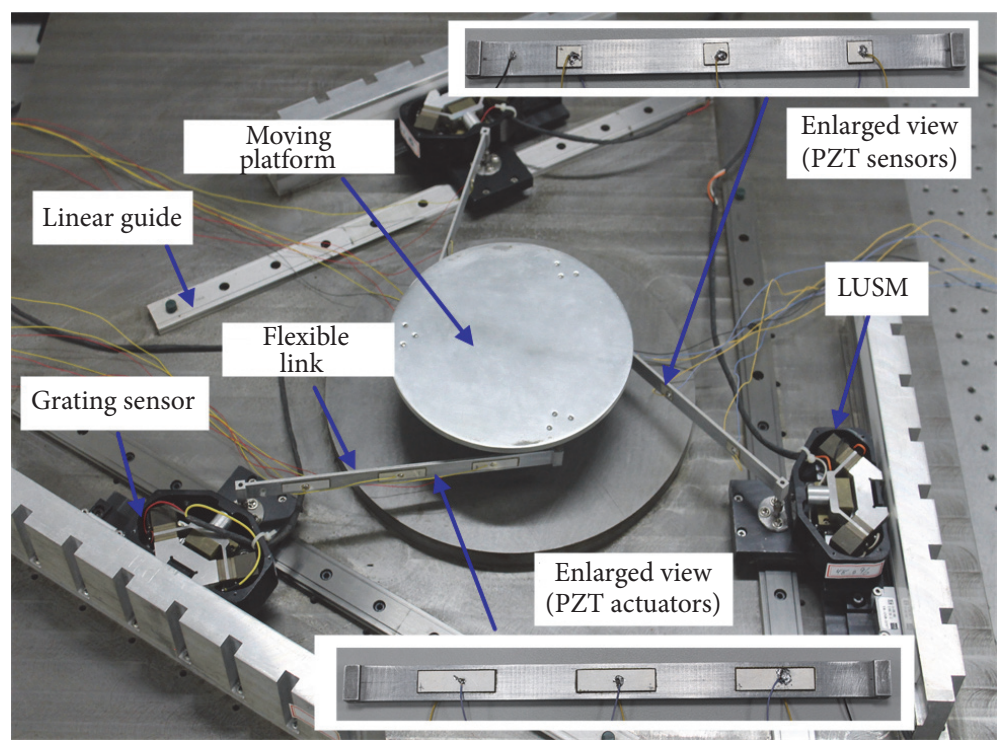

FIgURE 11: The layout of the PZT transducers and the setup of the experiment.

To verify the hybrid active vibration control method and the multimode vibration control ability, the first two modes of three flexible links are suppressed simultaneously. Before the experiments, the open-loop control test without the active vibration suppression is conducted to obtain the actual modal vibration energy $\left(E_{i j}=\omega_{i j}^{2} q_{i j}^{2}+\dot{q}_{i j}^{2}\right)$, and then the optimized feedback control gains for the first two modes are further derived based on (24):

$$
\begin{aligned}
& k_{d}^{11}: k_{d}^{12}=1: 0.312, \\
& k_{d}^{21}: k_{d}^{22}=1: 0.412, \\
& k_{d}^{31}: k_{d}^{32}=1: 0.355
\end{aligned}
$$

Since the unmodeled errors and parameter deviations are always existent in the theoretical model, the modal vibration 

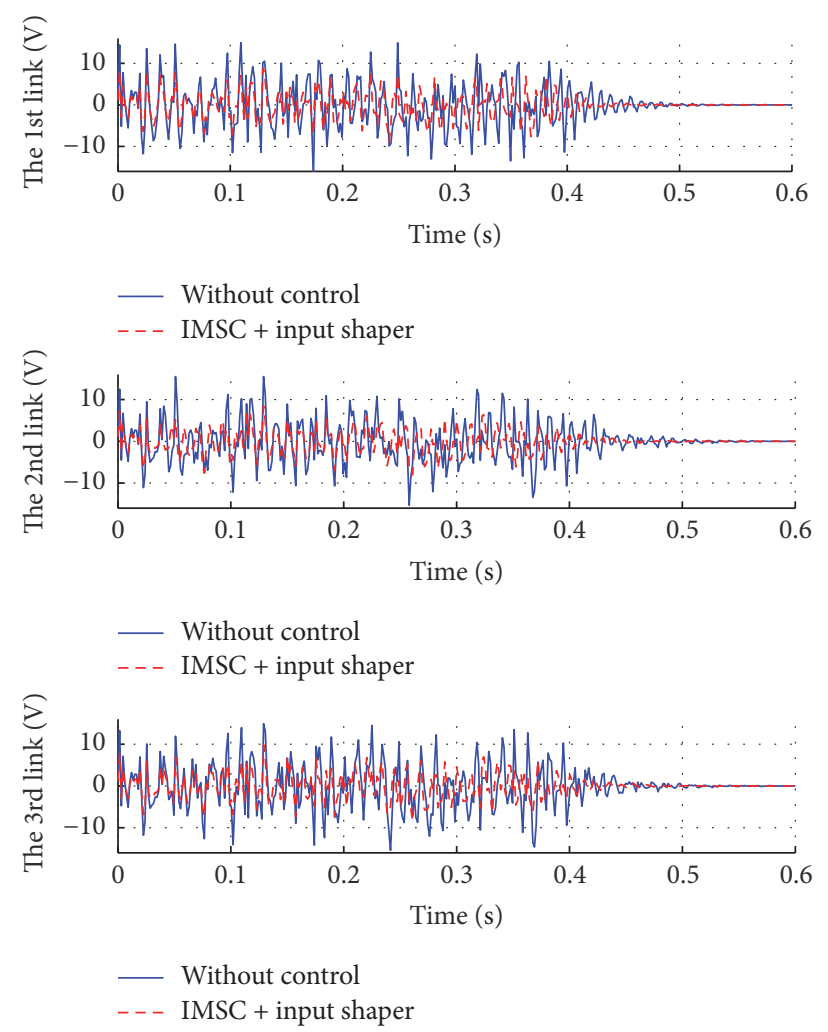

FIGURE 12: Response voltages of the PZT sensors at $150 \mathrm{~mm}$ of the three links.

energy calculated in simulation work is different from that obtained in the real-time test, and hence the optimized control gains in (27) are different from that calculated in simulation work (see (26)). With the optimized control gains, the response voltages on the three-quarters $(150 \mathrm{~mm})$ of the three flexible links are shown in Figure 12. It is clearly observed in Figure 12 that both the structural and the residual vibrations of the three flexible links are significantly suppressed using the hybrid active vibration control method. The residual vibrations are completely damped within $0.07 \mathrm{~s}$ which is almost 50\% faster than without control. Figures 13-16 are the power spectral density (PSD) of the first mode and second mode of the three flexible links extracted from the modal filters. With the IMSC method, the PSD of the first mode of the three links are reduced by $44.97 \%, 42.92 \%$, and $42.32 \%$, while those of the second mode are decreased by $48.33 \%$, $49.62 \%$, and $52.21 \%$, respectively. It is also found that in Figures 13-16 the first and second modes of the three flexible links have a little difference to those tested in the modal control experiments, for example, $92.5 \mathrm{~Hz}$ and $241.3 \mathrm{~Hz}$. These are mainly due to the geometrical error among the three links and the deviations introduced in the sensing and extracting procedures. However, due to the limitation of the number of the PZT pairs (in our experiments, only three PZT pairs are used), the independent vibration mode may not be separated from other modes completely, and hence we can find that the second natural mode around $240 \mathrm{~Hz}$ is also seen in the PSD plot of the first mode while the first natural mode around $92 \mathrm{~Hz}$ appears in the PSD plot of the second mode.
Besides, many other forced vibration frequencies are also illustrated in Figures 13-16, and these frequencies remain almost unchanged with the active vibration control. These forced vibration frequencies are likely from the different dynamics of each link, the different driving forces of the LUSM, and the coupling effect between the rigid motion and elastic motion. Therefore, the forced vibration frequencies observed in the first mode and second mode of the three flexible links are not identical and also not affected by the proposed vibration control since the frequencies of the forced vibration are far from the natural frequencies.

Similar to the analysis procedure in the simulation work, different control cases, namely, only controlling the first link and controlling the first and second link, are conducted as two comparison experiments to investigate the coupled vibration features among three flexible links. Since the first vibration mode is the dominant mode as the PSD of the first mode is much larger than the second mode, only the first mode is targeted to be controlled in the comparison cases. As shown in Figures 17 and 18, when only the first link is controlled, the PSD of the first mode of the first two links are reduced by $42.30 \%$ and $1.5 \%$, but the third link is increased by $0.92 \%$; when the first link and the second link are controlled, the PSD of the first mode of the three links are reduced by $43.62 \%, 43.23 \%$, and $1.84 \%$, respectively. The experiments results match well the simulation results (Figures 7 and 9) and further validate the effectiveness of the theoretical dynamic analysis. As analyzed in the simulation work, the elastic motion of the individual link is affected by other links 


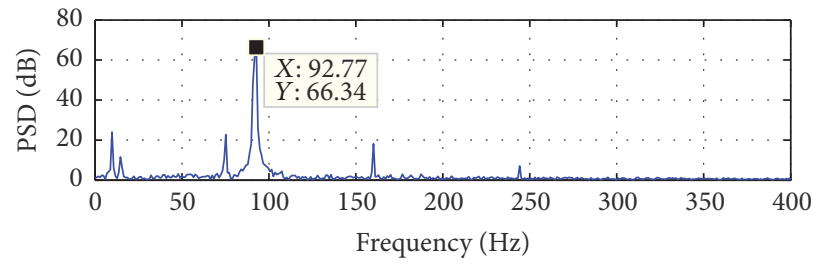

_ The 1st mode of the 1st link without control

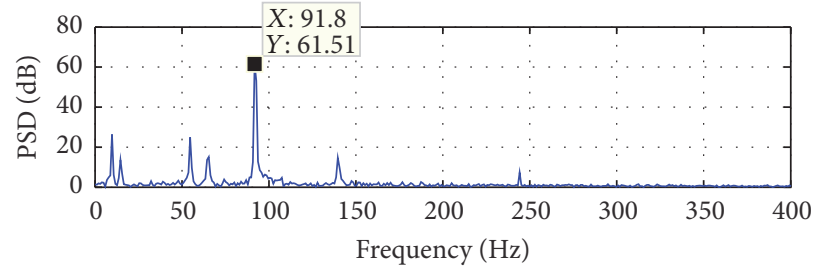

_ The 1st mode of the 2 nd link without control

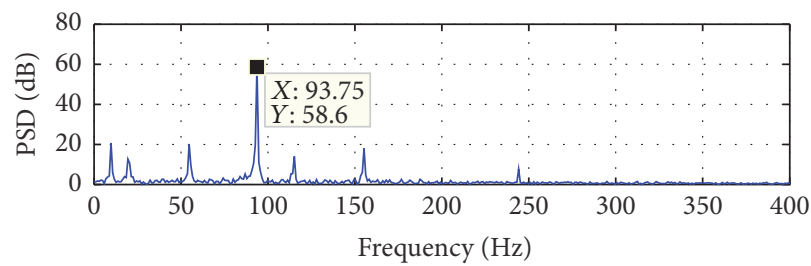

— The 1st mode of the 3rd link without control

FIGURE 13: The PSD plot of the first mode of the three links without control.

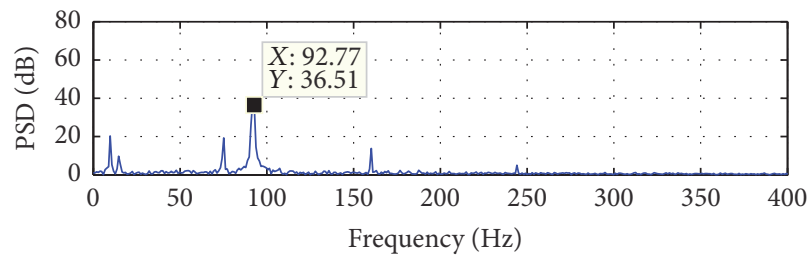

The 1st mode of the 1st link with three links controlled

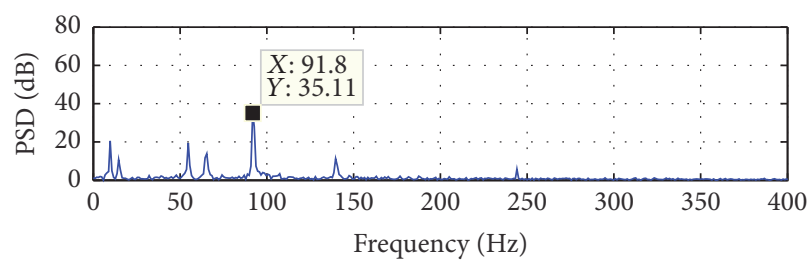

The 1st mode of the 2 nd link with three links controlled

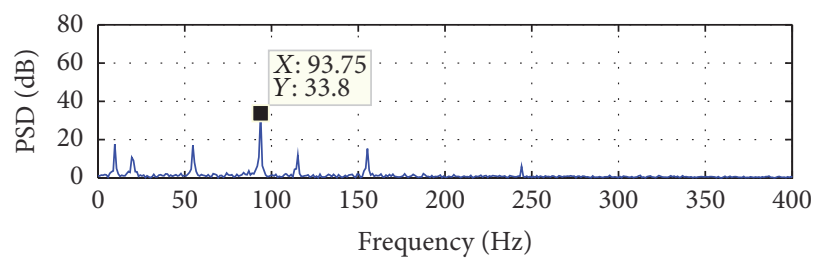

— The 1st mode of the 3rd link with three links controlled

FIGURE 14: The PSD plot of the first mode of the three links with control. 

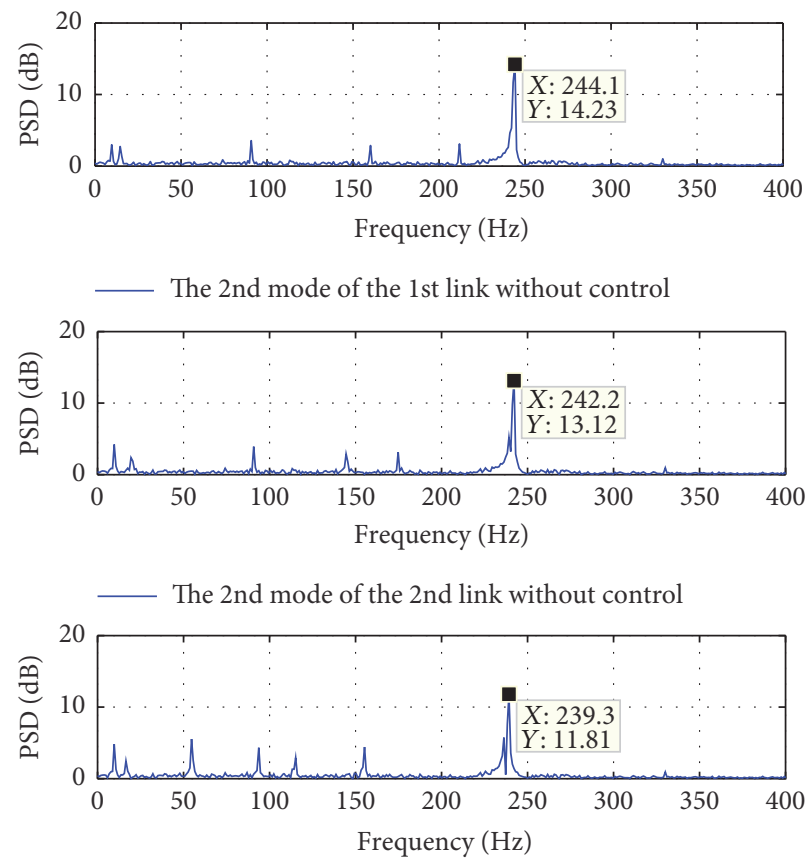

The 2nd mode of the 3rd link without control

FIGURE 15: The PSD plot of the second mode of the three links without control.

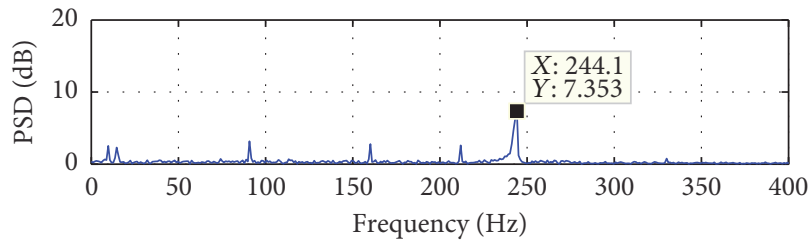

_ The 2nd mode of the 1st link with three links controlled
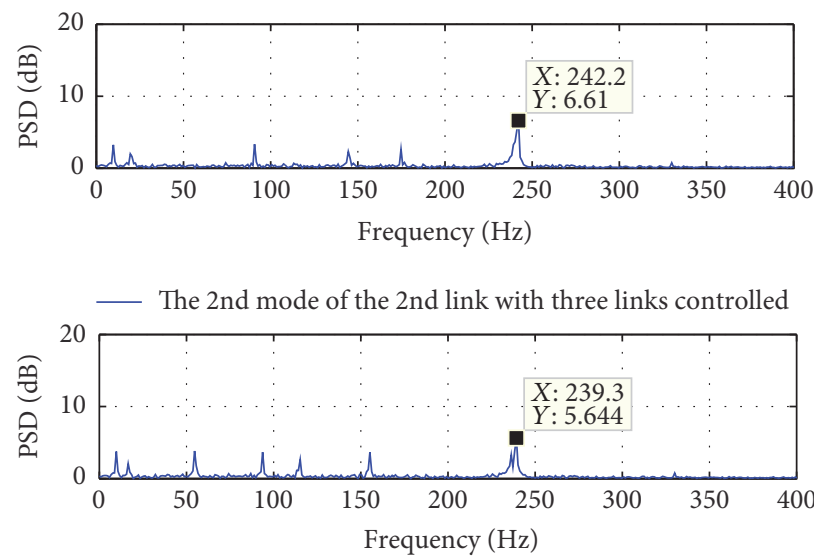

— The 2nd mode of the 3rd link with three links controlled

Figure 16: The PSD plot of the second mode of the three links with control.

through the coupled item $-C_{f f} \dot{\bar{q}}\left(\bar{q}_{1}, \bar{q}_{2}, \bar{q}_{3}, \dot{\bar{q}}_{1}, \dot{\bar{q}}_{2}, \dot{\bar{q}}_{3}\right)$ in (9). The vibration control results for the three different cases are summarized in Table 1, and the comparison results show that the more the links under control, the better the vibration suppression effect achieved. The control effects of the first mode of the first link are improved from $42.30 \%$ to $44.97 \%$ with the number of controlled links increased from 1 to 3 . The variation of the PSD of the third link is changed from positive $0.92 \%$ to negative $1.84 \%$ with the number of controlled links increased from 1 to 2 . 

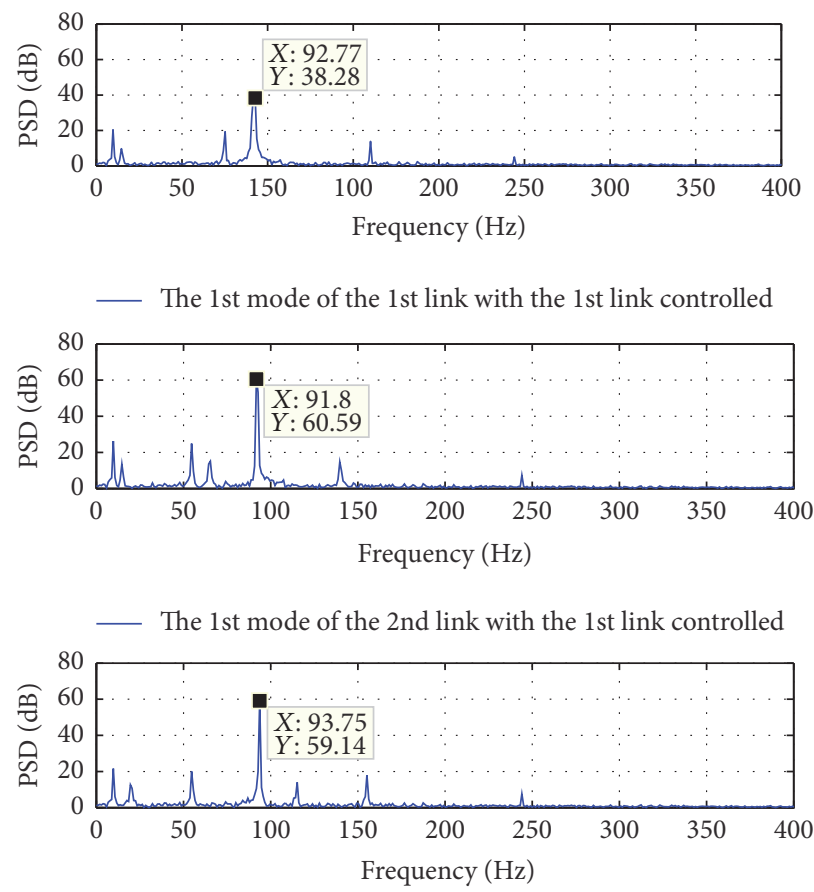

_ The 1st mode of the 3rd link with the 1st link controlled

Figure 17: The PSD plot of the first mode of the three links under the second case.

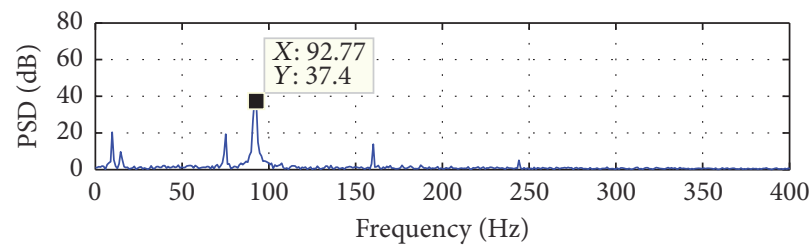

_ The 1st mode of the 1st link with the 1st and 2nd links controlled

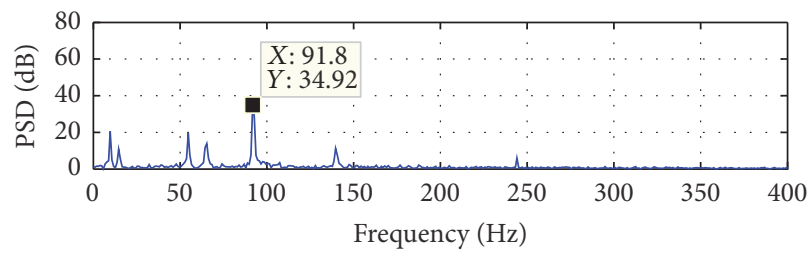

— The 1st mode of the 2nd link with the 1st and 2nd links controlled

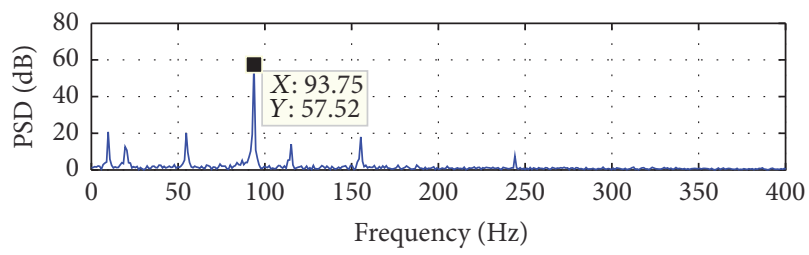

_ The 1st mode of the 3rd link with the 1st and 2nd links controlled

FIGURE 18: The PSD plot of the first mode of the three links under the third case.

However, in the control experiments, only the coupled features among the three flexible links are studied. The coupled relationship between the rigid and elastic motion is not experimentally validated due to the limitation of the capture frequencies of the charge coupled device (CCD) camera. In our experimental setup, the CCD camera with 40 frames per second is used to achieve the calibration work and the feedback position control of the moving platform, 
TABLE 1: The summarized results of the three comparison cases.

\begin{tabular}{lcccc}
\hline & Uncontrolled (PSD) & Three controlled (PSD) & The 1st and 2nd controlled (PSD) & The 1st controlled (PSD) \\
\hline 1st link & 66.34 & $36.51(-44.97 \%)$ & $37.40(-43.62 \%)$ & $38.28(-42.30 \%)$ \\
2nd link & 61.51 & $35.11(-42.92 \%)$ & $34.92(-43.23 \%)$ & $60.59(-1.50 \%)$ \\
3rd link & 58.60 & $33.80(-42.32 \%)$ & $57.52(-1.84 \%)$ & $59.14(+0.92 \%)$ \\
\hline
\end{tabular}

but it is difficult to detect the residual vibration caused by the flexible links whose vibration frequencies are more than $90 \mathrm{~Hz}$. Other sensing instruments, such as accelerometers, lasers, or high frequency CCD, may be adopted to detect the residual oscillation of the moving platform in the future study.

\section{Conclusions}

This paper addresses active vibration control and coupled vibration characteristics analysis of a parallel manipulator with three flexible links. The one-pass method which considered the fully coupled dynamics features between the rigid and elastic motions is adopted based on Lagrange's multipliers and the AMM method. With multiple piezoelectric transducers integrated on the flexible links, the IMSC method combined with input shaper is developed to suppress multimode vibration of the flexible links in order to prevent the spillover phenomena. Computer simulation and experiments are carried out to verify the proposed hybrid active vibration control method, and the results show that both the structural and the residual vibrations of the three flexible links are all significantly damped. The coupled vibration features among three flexible links and the relationship between rigid and elastic motions are theoretically analyzed based on the simulation and experimental results. The experimental results show that the vibration control effects improved with the number of controlled links increased. In the near future, more different control cases, such as only controlling the second link or controlling the second and third link, may be conducted to further investigate the coupled features among the three flexible links.

\section{Competing Interests}

The authors declare that there are no competing interests regarding the publication of this paper.

\section{Acknowledgments}

This work was supported by the National Natural Science Foundation of China (Grants nos. 51605271, 51577112, 51375225, and 51305248) and Shanghai Training and Support Program for University Youth Teachers (ZZSD15084).

\section{References}

[1] Q. Zhang, J. K. Mills, W. L. Cleghorn, J. Jin, and C. Zhao, "Trajectory tracking and vibration suppression of a 3-PRR parallel manipulator with flexible links," Multibody System Dynamics, vol. 33, no. 1, pp. 27-60, 2015.
[2] O. Abdeljaber, O. Avci, and D. J. Inman, "Active vibration control of flexible cantilever plates using piezoelectric materials and artificial neural networks," Journal of Sound and Vibration, vol. 363, pp. 33-53, 2016.

[3] I. Bruant, L. Gallimard, and S. Nikoukar, "Optimal piezoelectric actuator and sensor location for active vibration control, using genetic algorithm," Journal of Sound and Vibration, vol. 329, no. 10, pp. 1615-1635, 2010.

[4] N. C. Singer, Residual Vibration Reduction in Computer Controlled Machines, Massachusetts Institute of Technology, Cambridge, Mass, USA, 1988.

[5] E. Pereira, J. R. Trapero, I. M. Díaz, and V. Feliu, "Adaptive input shaping for single-link flexible manipulators using an algebraic identification," Control Engineering Practice, vol. 20, no. 2, pp. 138-147, 2012.

[6] R. R. Orszulik and J. Shan, "Vibration control using input shaping and adaptive positive position feedback," Journal of Guidance, Control, and Dynamics, vol. 34, no. 4, pp. 1031-1044, 2011.

[7] M. O. T. Cole and T. Wongratanaphisan, "Optimal FIR input shaper designs for motion control with zero residual vibration," Journal of Dynamic Systems, Measurement and Control, vol. 133, no. 2, Article ID 021008, 2011.

[8] H. Deng, J.-D. Sun, S.-D. Huang, and G.-Z. Cao, "Vibration suppression of the flexible manipulator using optimal input shaper and linear quadratic regulator," in Proceedings of the 12th International Conference on Ubiquitous Robots and Ambient Intelligence (URAI '15), pp. 255-260, IEEE, Goyang, Republic of Korea, October 2015.

[9] Z. Y. Chu and J. Cui, "Experiment on vibration control of a two-link flexible manipulator using an input shaper and adaptive positive position feedback," Advances in Mechanical Engineering, vol. 7, no. 10, pp. 1-13, 2015.

[10] K. Kozak, I. Ebert-Uphoff, and W. Singhose, "Locally linearized dynamic analysis of parallel manipulators and application of input shaping to reduce vibrations," Journal of Mechanical Design, vol. 126, no. 1, pp. 156-168, 2004.

[11] B. Li, X. Zhang, J. K. Mills et al., "Vibration suppression of a 3-PRR flexible parallel manipulator using input shaping," in Proceedings of the International Conference on IEEE Mechatronics and Automation (ICMA '09), pp. 3539-3544, Changchun, China, August 2009.

[12] Q. Zhang, J. K. Mills, W. L. Cleghorn, J. Jin, and Z. Sun, "Dynamic model and input shaping control of a flexible link parallel manipulator considering the exact boundary conditions," Robotica, vol. 33, no. 6, pp. 1201-1230, 2015.

[13] E. Omidi and S. N. Mahmoodi, "Vibration control of collocated smart structures using $\mathrm{H} \infty$ modified positive position and velocity feedback," Journal of Vibration and Control, vol. 22, no. 10, pp. 2434-2442, 2016.

[14] S. Q. Zhang, H. N. Li, R. Schmidt, and P. C. Müller, "Disturbance rejection control for vibration suppression of piezoelectric laminated thin-walled structures," Journal of Sound and Vibration, vol. 333, no. 5, pp. 1209-1223, 2014. 
[15] X. Zhang, J. K. Mills, and W. L. Cleghorn, "Multi-mode vibration control and position error analysis of parallel manipulator with multiple flexible links," Transactions of the Canadian Society for Mechanical Engineering, vol. 34, no. 2, pp. 197-213, 2010.

[16] D. J. Inman, "Active modal control for smart structures," Philosophical Transactions of the Royal Society A: Mathematical, Physical and Engineering Sciences, vol. 359, no. 1778, pp. 205-219, 2001.

[17] L. Meirovitch and H. Baruh, "Optimal control of damped flexible gyroscopic systems," Journal of Guidance, Control, and Dynamics, vol. 4, no. 2, pp. 157-163, 1981.

[18] A. Baz and S. Poh, "Performance of an active control system with piezoelectric actuators," Journal of Sound and Vibration, vol. 126, no. 2, pp. 327-343, 1988.

[19] S. P. Singh, H. Singh Pruthi, and V. P. Agarwal, "Efficient modal control strategies for active control of vibrations," Journal of Sound and Vibration, vol. 262, no. 3, pp. 563-575, 2003.

[20] Q. Zhang, J. Jin, J. Zhang, and C. Zhao, "Active vibration suppression of a 3-DOF flexible parallel manipulator using efficient modal control," Shock and Vibration, vol. 2014, Article ID 953694, 10 pages, 2014.

[21] D. Wu, L. Huang, B. Pan, Y. Wang, and S. Wu, "Experimental study and numerical simulation of active vibration control of a highly flexible beam using piezoelectric intelligent material," Aerospace Science and Technology, vol. 37, pp. 10-19, 2014.

[22] F. Braghin, S. Cinquemani, and F. Resta, "A new approach to the synthesis of modal control laws in active structural vibration control," Journal of Vibration and Control, vol. 19, no. 2, pp. 163$182,2013$.

[23] G. Bagordo, G. Cazzulani, F. Resta, and F. Ripamonti, "A modal disturbance estimator for vibration suppression in nonlinear flexible structures," Journal of Sound and Vibration, vol. 330, no. 25, pp. 6061-6069, 2011.

[24] F. Resta, F. Ripamonti, G. Cazzulani, and M. Ferrari, "Independent modal control for nonlinear flexible structures: an experimental test rig," Journal of Sound and Vibration, vol. 329, no. 8, pp. 961-972, 2010.

[25] X. Guo and J. Jiang, "Optimization of actuator placement in a truss-cored sandwich plate with independent modal space control," Smart Materials and Structures, vol. 20, no. 11, Article ID 115011, 2011.

[26] X. Zhang, J. K. Mills, and W. L. Cleghorn, "Coupling characteristics of rigid body motion and elastic deformation of a 3PRR parallel manipulator with flexible links," Multibody System Dynamics, vol. 21, no. 2, pp. 167-192, 2009.

[27] W. E. Singhose and N. C. Singer, "Effects of input shaping on two-dimensional trajectory following," IEEE Transactions on Robotics and Automation, vol. 12, no. 6, pp. 881-887, 1996.

[28] N. C. Singer, Residual vibration reduction in computer controlled machines [Ph.D. dissertation], Massachusetts Institute of Technology, Cambridge, Mass, USA, 1989.

[29] N. Jalili, Piezoelectric-Based Vibration Control: From Macro to Micro/Nano Scale Systems, Springer, Berlin, Germany, 2010. 


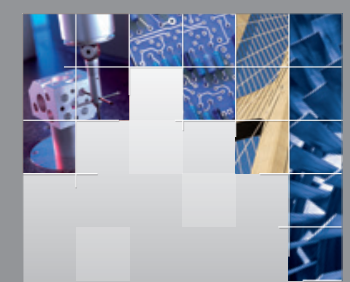

\section{Enfincering}
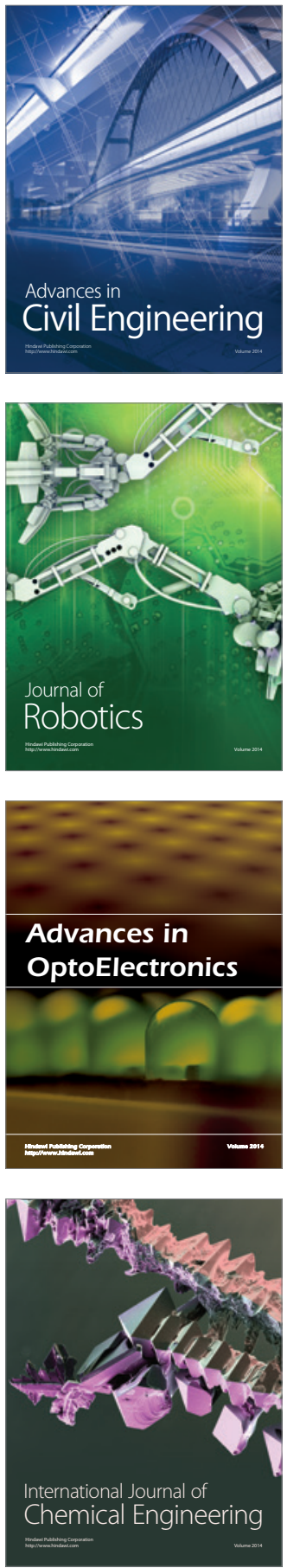

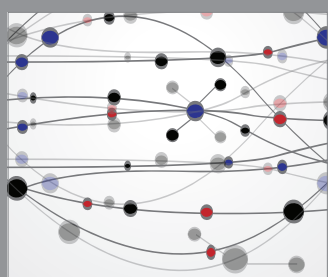

The Scientific World Journal

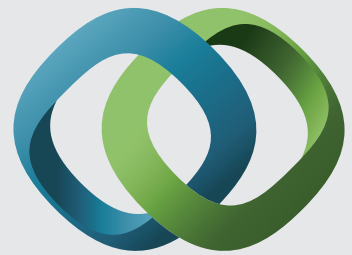

\section{Hindawi}

Submit your manuscripts at

http://www.hindawi.com
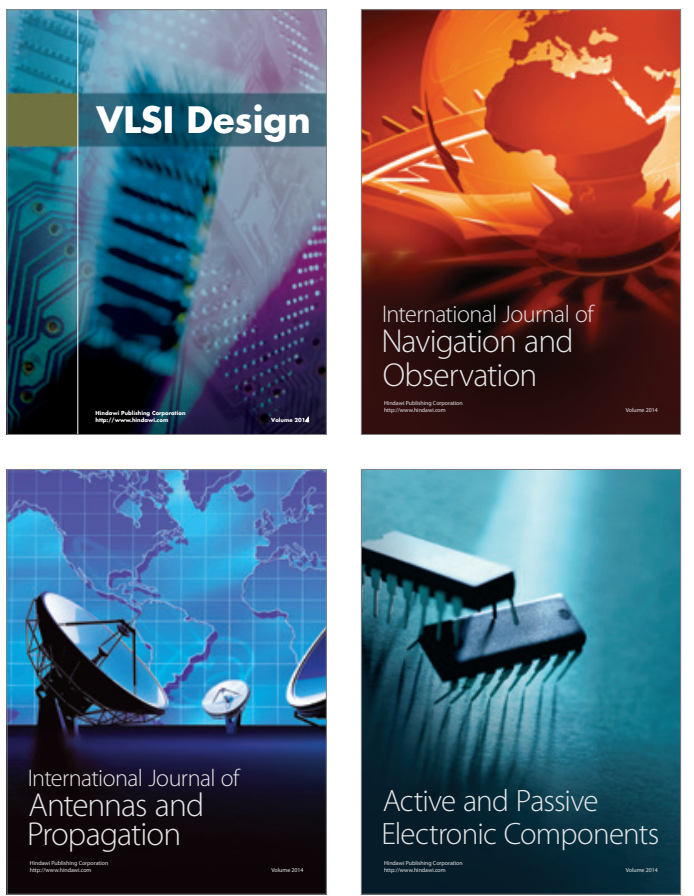
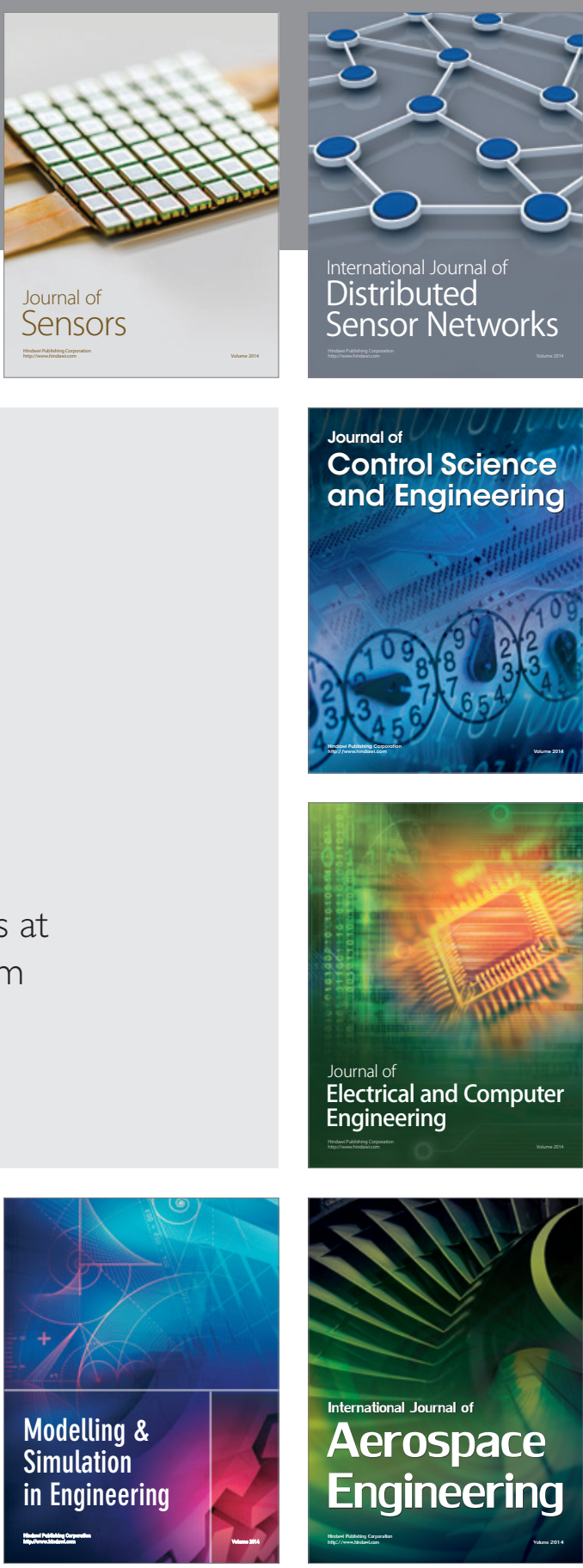

International Journal of

Distributed

Sensor Networks

Journal of

Control Science

and Engineering
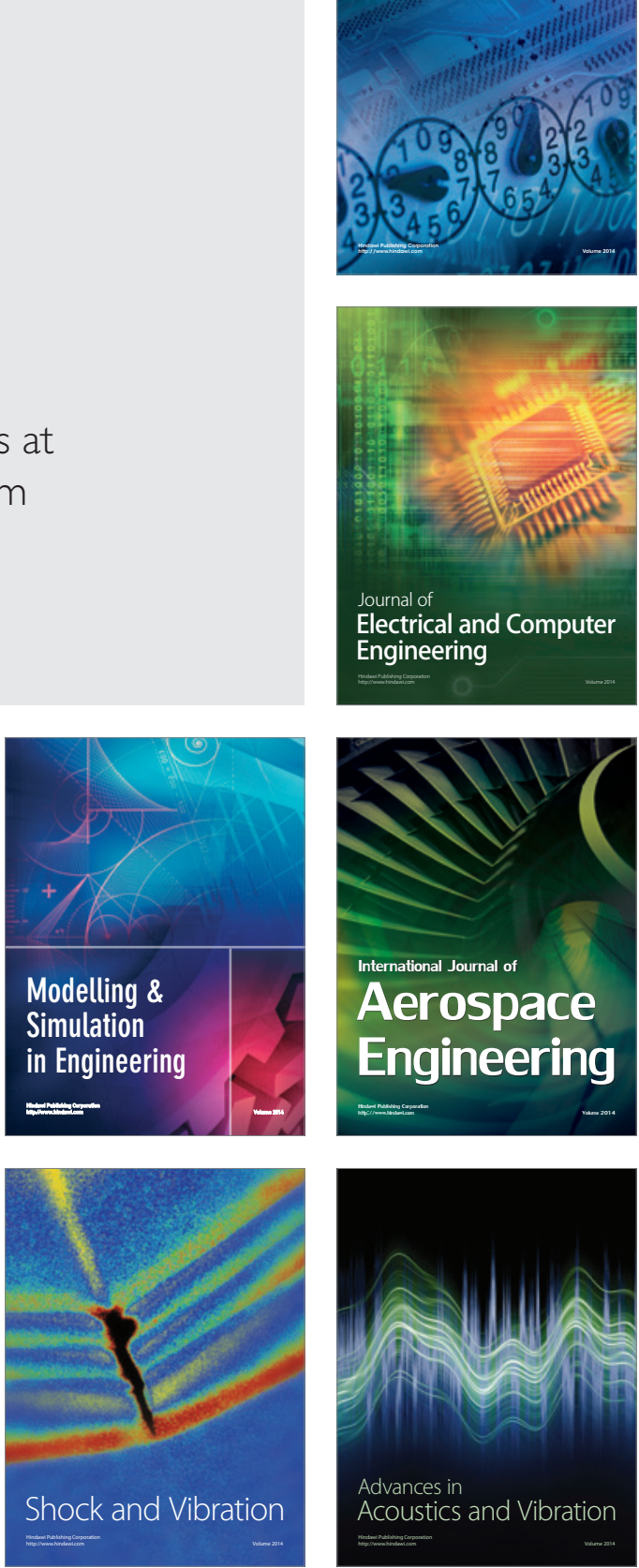